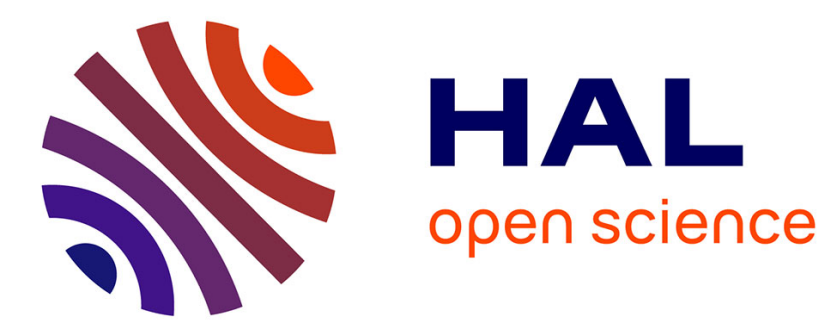

\title{
SmEdA vibro-acoustic modelling in the mid-frequency range including the effect of dissipative treatments
}

Ha Dong Hwang, Laurent Maxit, Kerem Ege, Youssef Gerges, Jean-Louis Guyader

\section{- To cite this version:}

Ha Dong Hwang, Laurent Maxit, Kerem Ege, Youssef Gerges, Jean-Louis Guyader. SmEdA vibroacoustic modelling in the mid-frequency range including the effect of dissipative treatments. Journal of Sound and Vibration, 2017, 393, pp.187-215. 10.1016/j.jsv.2017.01.024 . hal-01444453

\section{HAL Id: hal-01444453 \\ https://hal.science/hal-01444453}

Submitted on 25 Jan 2017

HAL is a multi-disciplinary open access archive for the deposit and dissemination of scientific research documents, whether they are published or not. The documents may come from teaching and research institutions in France or abroad, or from public or private research centers.
L'archive ouverte pluridisciplinaire HAL, est destinée au dépôt et à la diffusion de documents scientifiques de niveau recherche, publiés ou non, émanant des établissements d'enseignement et de recherche français ou étrangers, des laboratoires publics ou privés. 


\title{
SmEdA vibro-acoustic modelling in the mid-frequency range including the effect of dissipative treatments
}

\author{
H. D. Hwang, L. Maxit*, K. Ege, Y. Gerges, J.-L. Guyader \\ Univ Lyon, INSA-Lyon, LVA EA677, 25 bis avenue Jean Capelle, \\ F-69621 Villeurbanne Cedex, France
}

\begin{abstract}
Vibro-acoustic simulation in the mid-frequency range is of interest for automotive and truck constructors. The dissipative treatments used for noise and vibration control such as viscoelastic patches and acoustic absorbing materials must be taken into account in the problem. The Statistical modal Energy distribution Analysis (SmEdA) model consists in extending Statistical Energy Analysis (SEA) to the mid-frequency range by establishing power balance equations between the modes of the different subsystems. The modal basis of uncoupled-subsystems that can be estimated by the finite element method in the midfrequency range is used as input data. SmEdA was originally developed by considering constant modal damping factors for each subsystem. However, this means that it cannot describe the local distribution of dissipative materials. To overcome this issue, a methodology is proposed here to take into account the effect of these materials. This methodology is based on the finite element models of the subsystems that include well-known homogenized material models of dissipative treatments. The Galerkin method with subsystem normal modes is used to estimate the modal damping loss factors. Cross-modal coupling terms which appear in the formulation due to the dissipative materials are assumed to be negligible. An approximation of the energy sharing between the subsystems damped by
\end{abstract}


dissipative materials is then described by SmEdA. The different steps of the method are validated experimentally by applying it to a laboratory test case composed of a plate-cavity system with different configurations of dissipative treatments. The comparison between the experimental and the simulation results shows good agreement in the mid-frequency range.

Key words: vibro-acoustic modelling, fluid-structure interaction, mid-frequency analysis, viscoelastic layer, porous materials, modal damping loss factor

\section{Nomenclature}

$a_{m} \quad$ the amplitude of the $m^{t h}$ panel mode (dimensionless quantity)

$b_{n} \quad$ the amplitude of the $n^{\text {th }}$ cavity mode (dimensionless quantity)

$c_{n} \quad$ the integrated amplitude of the $n^{\text {th }}$ cavity mode (s)

$c_{\text {air }} \quad$ the speed of sound in the air $\left(\mathrm{m} \mathrm{s}^{-1}\right)$

C the fluid-structure coupling matrix $\left(\mathrm{m}^{2}\right)$

$E_{\mathrm{ba}}^{*} \quad$ the complex Young's modulus of the bare plate $\left(\mathrm{N} \mathrm{m}^{-\mathbf{2}}\right)$

$E_{\mathrm{eq}, \mathrm{m}}^{*}$ the complex equivalent Young's modulus of the multilayer $\left(\mathbf{N ~ m}^{-\mathbf{2}}\right)$

$E_{m} \quad$ the energy of the $m^{t h}$ panel mode $(\mathbf{J})$

$E_{n} \quad$ the energy of the $n^{\text {th }}$ cavity mode $(\mathbf{J})$

$E_{1} \quad$ the total energy of the plate in a third octave band $(\mathbf{J})$

$E_{2} \quad$ the total energy inside the cavity in a third octave band (J)

F the panel nodal force vector $\left(\mathbf{N}\right.$ or $\left.\mathbf{k g ~} \mathbf{~ m ~ s}^{-\mathbf{2}}\right)$

$F_{m} \quad$ the generalized force of the $m^{\text {th }}$ panel mode $(\mathbf{N} \mathbf{~ m})$

$F_{0}(t)$ the amplitude of the external force in function of time $t(\mathbf{N})$

$h \quad$ the thickness of the bared plate (m)

$h_{i} \quad$ the thickness of the $i^{t h}$ layer (m)

Email address: laurent maxiteinsa-lyon. fr (H. D. Hwang, L. Maxit*, K. Ege, Y. Gerges, J.-L. Guyader). 
$K_{\text {air }}^{*} \quad$ the complex compressibility of the air $\left(\mathrm{N} \mathrm{m}^{-\mathbf{2}}\right)$

$\overline{\mathbf{K}}_{\text {air }}$ the kinetic energy matrix of the 'undamped' air domain $\left(\mathrm{kg}^{-\mathbf{1}} \mathbf{m}^{\mathbf{4}}\right)$

$\mathbf{K}_{\mathrm{ba}}^{*} \quad$ the complex stiffness matrix of the 'damped' bare panel $\left(\mathbf{N ~ m}^{-\mathbf{1}}\right)$

$\mathbf{K}_{\text {air }}^{e} \quad$ the elementary kinetic energy matrix of the 'undamped' air domain ( $\left.\mathbf{m}\right)$

$\overline{\mathbf{K}}_{\mathrm{ba}} \quad$ the stiffness matrix of the 'undamped' bare panel $\left(\mathbf{N ~ m}^{-\mathbf{1}}\right)$

$\mathbf{K}_{\mathrm{ba}}^{e} \quad$ the elementary stiffness matrix of the 'undamped' bare panel (m)

$K_{\text {eq,f }}^{*}$ the complex equivalent homogenized fluid compressibility $\left(\mathrm{N} \mathrm{m}^{-\mathbf{2}}\right)$

$\mathbf{K}_{\mathrm{c}} \quad$ the global kinetic energy matrix of the cavity $\left(\mathrm{kg}^{-1} \mathbf{m}^{4}\right)$

$\mathbf{K}_{\text {eq,f }}^{*}$ the complex kinetic energy matrix of the 'damped' equivalent fluid domain $\left(\mathrm{kg}^{-1} \mathrm{~m}^{4}\right)$

$\overline{\mathbf{K}}_{\text {eq,f }}$ the kinetic energy matrix of the 'undamped' equivalent fluid domain $\left(\mathrm{kg}^{-1} \mathbf{m}^{4}\right)$

$\mathbf{K}_{\mathrm{eq}, \mathrm{f}}^{e}$ the elementary kinetic energy matrix of the 'undamped' equivalent fluid domain $(\mathbf{m})$

$\mathbf{K}_{\mathrm{eq}, \mathrm{m}}^{*}$ the complex stiffness matrix of the 'damped' equivalent multilayer domain $\left(\mathrm{N} \mathrm{m}^{-1}\right)$

$\overline{\mathbf{K}}_{\text {eq,m }}$ the stiffness matrix of the 'undamped' equivalent multilayer domain $\left(\mathbf{N ~ m}^{-\mathbf{1}}\right)$

$\mathbf{K}_{\mathrm{eq}, \mathrm{m}}^{e}$ the elementary stiffness matrix of the 'undamped' equivalent multilayer domain (m)

$\mathbf{K}_{\mathrm{s}}$ the global stiffness matrix of the panel $\left(\mathrm{N} \mathrm{m}^{-\mathbf{1}}\right.$ or $\left.\mathrm{kg} \mathrm{s}^{-\mathbf{2}}\right)$

$L_{x} \quad$ the dimension of the plate/cavity in the $x$ direction ( $\left.\mathbf{m}\right)$

$L_{y} \quad$ the dimension of the plate/cavity in the $y$ direction (m)

$L_{z} \quad$ the dimension of the cavity in the $z$ direction $(\mathbf{m})$

$\widetilde{M} \quad$ the set of the panel resonant modes (dimensionless quantity)

$\mathbf{M}_{\text {air }}^{*}$ the complex strain energy matrix of the 'damped' air domain $\left(\mathrm{kg}^{-1} \mathbf{m}^{4} \mathbf{s}^{2}\right)$

$\overline{\mathbf{M}}_{\text {air }}$ the strain energy matrix of the 'undamped' air domain $\left(\mathrm{kg}^{-1} \mathbf{m}^{4} \mathbf{s}^{\mathbf{2}}\right)$

$\mathbf{M}_{\text {air }}^{e} \quad$ the elementary strain energy matrix of the 'undamped' air domain $\left(\mathbf{m}^{\mathbf{3}}\right)$

$\mathrm{M}_{\mathrm{ba}}$ the mass matrix of the bared plate $(\mathrm{kg})$ 
$M_{c} \quad$ the global strain energy matrix of the cavity $\left(\mathrm{kg}^{-1} \mathbf{m}^{4} \mathbf{s}^{2}\right)$

$M_{e} \quad$ the excitation point

$\mathbf{M}_{\text {eq,f }}^{*}$ the complex strain energy matrix of the 'damped' equivalent fluid domain $\left(\mathrm{kg}^{-1} \mathrm{~m}^{4} \mathrm{~s}^{2}\right)$

$\overline{\mathbf{M}}_{\mathrm{eq}, \mathrm{f}}$ the strain energy matrix of the 'undamped' equivalent fluid domain $\left(\mathrm{kg}^{-1} \mathbf{m}^{4} \mathbf{s}^{2}\right)$

$\mathbf{M}_{\mathrm{eq}, \mathrm{f}}^{e}$ the elementary strain energy matrix of the 'undamped' equivalent fluid domain $\left(\mathbf{m}^{3}\right)$

$\mathbf{M}_{\mathrm{eq}, \mathrm{m}}$ the mass matrix of the equivalent multilayer domain $(\mathrm{kg})$

$M_{m} \quad$ the generalized mass of the $m^{\text {th }}$ panel mode $\left(\mathrm{kg} \mathrm{m}^{2}\right)$

$M_{n} \quad$ the generalized 'mass' of the $n^{\text {th }}$ cavity mode $\left(\mathrm{kg} \mathrm{m}^{2} \mathbf{s}^{-2}\right)$

$\mathbf{M}_{\mathrm{s}}$ the global mass matrix of the panel $(\mathrm{kg})$

$\widetilde{N} \quad$ the set of the cavity resonant modes (dimensionless quantity)

$P \quad$ the acoustic pressure $\left(\mathrm{N} \mathrm{m}^{-\mathbf{2}}\right)$

$\mathbf{P} \quad$ the nodal pressure vector of the cavity $\left(\mathrm{N} \mathrm{m}^{-\mathbf{2}}\right)$

$\bar{p}_{i}^{2} \quad$ the spectrum of the space-averaged quadratic pressure inside the cavity $\left(\mathbf{N}^{2} \mathbf{m}^{-4} \mathbf{s}\right)$

$p_{n}^{i} \quad$ the modal pressure at node $i$ of the $n^{t h}$ mode $\left(\mathbf{N ~ m}^{-\mathbf{2}}\right)$

$\mathbf{P}_{\mathbf{n}} \quad$ the nodal pressure vector of the $n^{\text {th }}$ cavity mode $\left(\mathbf{N ~ m}^{-\mathbf{2}}\right)$

$S_{F_{0} F_{0}}$ the power spectrum density of the excited force $\left(\mathbf{N}^{2} \mathbf{s}\right)$

$s^{i} \quad$ the elementary surface attributed to node $i\left(\mathbf{m}^{\mathbf{2}}\right)$

$\bar{v}_{i}^{2} \quad$ the spectrum of the space-averaged quadratic velocity of the plate $\left(\mathbf{m}^{2} \mathbf{s}^{-1}\right)$

W the nodal displacement vector of the panel (m)

$\mathbf{W}_{\mathbf{m}}$ the nodal displacement vector of the $m^{\text {th }}$ panel mode (m)

$w_{m}^{i} \quad$ the normal displacement at node $i$ of the $m^{t h}$ mode ( $\left.\mathbf{m}\right)$

$W_{m n}$ the intermodal work between mode $m$ and mode $n\left(\mathbf{J}\right.$ or $\mathbf{N} \mathbf{m}$ or $\left.\mathbf{k g} \mathbf{~ m}^{\mathbf{2}} \mathbf{s}^{-\mathbf{2}}\right)$

$Z_{c}^{*} \quad$ the characteristic impedance of the absorbent material $\left(\mathrm{kg} \mathrm{s}^{-\mathbf{1}} \mathbf{m}^{-\mathbf{2}}\right)$

$\beta_{m n} \quad$ the intermodal coupling factor between the mode $m$ and the mode $n\left(\mathbf{s}^{-\mathbf{1}}\right)$

$\beta_{m n}^{\omega}$ the spectral intermodal coupling factor between the mode $m$ and the mode 
$n$ (s)

$\beta_{m n}^{W}$ the spatial intermodal coupling factor between the mode $m$ and the mode $n$ $\left(\mathrm{s}^{-2}\right)$

$\delta f \quad$ the frequency resolution $\left(\mathbf{s}^{-1}\right)$

$\Delta \omega \quad$ the width of the angular frequency band of interest $\left(\mathbf{s}^{-1}\right)$

$\eta_{m} \quad$ the damping loss factor of the $m^{\text {th }}$ panel mode (dimensionless quantity)

$\eta_{n} \quad$ the damping loss factor of the $n^{\text {th }}$ cavity mode ( dimensionless quantity)

$\eta_{\text {air }}$ the damping loss factor of the air (dimensionless quantity)

$\eta_{b a}$ the damping loss factor of the bared structure (dimensionless quantity)

$\eta_{e q, m}$ the equivalent damping loss factor of the multilayer (dimensionless quantity)

$\eta_{e q, f}$ the damping loss factor of the equivalent fluid due to thermal effect (dimensionless quantity)

$\eta^{1} \quad$ the global damping loss factor of the structure (dimensionless quantity)

$\eta^{2} \quad$ the global damping loss factor of the cavity (dimensionless quantity)

$v_{\text {eq,m }}$ the equivalent Poisson's ratio of the multilayer (dimensionless quantity)

$v_{i} \quad$ the Poisson's ratio of the $i^{t h}$ layer (dimensionless quantity)

$\Pi_{m}^{\text {diss }} \quad$ the time-averaged power dissipated by the internal damping of mode $m$ $\left(\mathrm{J} \mathrm{s}^{-1}\right)$

$\Pi_{m}^{\mathrm{inj}} \quad$ the time-averaged power injected by the generalized force $F_{m}\left(\mathbf{J ~ s}^{-\mathbf{1}}\right)$

$\Pi_{\mathrm{mn}} \quad$ the time-averaged power flow exchanged between the mode $m$ and the mode $n\left(\mathbf{J ~ s}^{-\mathbf{1}}\right)$

$\rho \quad$ the density of the bared plate $\left(\mathrm{kg} \mathrm{m}^{-3}\right)$

$\rho_{\text {air }} \quad$ the density of the air $\left(\mathrm{kg} \mathrm{m}^{-3}\right)$

$\rho_{\mathrm{eq}, \mathrm{f}}^{*} \quad$ the complex equivalent fluid density of the absorbent $\left(\mathrm{kg} \mathrm{m}^{-\mathbf{3}}\right)$

$\rho_{\text {eq,m }}$ the equivalent density of the multilayer $\left(\mathrm{kg} \mathrm{m}^{-3}\right)$

$\chi_{\text {eq,f }}$ the damping loss factor of the equivalent fluid due to viscous effect (dimen- 


sionless quantity)
$\omega \quad$ the angular frequency $\left(\mathbf{s}^{-1}\right)$
$\omega_{c} \quad$ the central angular frequency of the frequency band of interest $\left(\mathbf{s}^{-1}\right)$
$\omega_{m} \quad$ the angular frequency of the $m^{t h}$ panel mode $\left(\mathbf{s}^{-1}\right)$
$\omega_{n} \quad$ the angular frequency of the $n^{t h}$ cavity mode $\left(\mathbf{s}^{-1}\right)$
$\bar{\omega}_{n} \quad$ the modified angular frequency of the $n^{t h}$ cavity mode $\left(\mathbf{s}^{-1}\right)$

\section{Introduction}

The noise, vibration and harshness (NVH) performance of modern vehicles is of great importance for manufacturers in globally competitive markets. In order to control this performance at the design stage of a new vehicle, engineers must rely on accurate predictive vibro-acoustic tools. These tools should cover a large part of the audible frequency range $[1-3]$ without being limited to modelling the behaviour of the body-in-white structure only. Indeed, to be relevant, these tools must take into account the effects of dissipative treatments such as viscoelastic layers and poroelastic materials.

In the low frequency range, the Finite Element Method (FEM) [4] is generally used due to its considerable versatility. Various formulations have been implemented to capture the effects of dissipative materials. For instance, viscoelastic treatments can be represented using classical lamination theory [5] whereas porous materials can be represented by Biot's model [6,7] or the simplified equivalent fluid model [8].

On the other hand, for high frequencies, Statistical Energy Analysis (SEA) [9-11] is commonly considered by industrial companies because it gives quick and accurate assessments of the performance of sound packages [1, 12]. SEA parameters are generally estimated with dedicated models of the parts of the system which include 
dissipative materials. For instance, the transmission loss of a multi-layered noise control treatment can be predicted using the transfer matrix method [12,13] and the equivalent damping loss factors of the cavity with noise control treatments can be deduced. These parameters are then used in the SEA model of the global system.

The main drawback of these two methods (i.e. FEM, SEA) is that they do not generally cover the whole frequency band of interest. FEM has proved its efficiency and relevance for analyzing the modal behaviour of complex mechanical structures and their sound radiations. Beyond this frequency range, this approach is computationally costly and can be problematic for describing the variabilities and uncertainties of the system considered. Moreover, analyzing the considerable amount of results to extract the most predominant phenomena involved in noise radiation is not an easy task. Furthermore, SEA relies on numerous assumptions that limit its application to high frequencies. In particular, each subsystem must present a high number of resonant modes per frequency band of analysis and a high modal overlap (typically, greater than one) [14,15]. The development of dedicated methods for the mid-frequency range to fill this frequency gap in modelling capability remains a hot topic of research. Recent research projects have been carried out that include dissipative treatments in their mid-frequency models such as the Wave Based Method [16-18] and the hybrid FE-SEA [19-22].

In this context, the present work proposes a methodology for including dissipative materials in a Statistical modal Energy distribution Analysis (SmEdA) model [2325]. This method extends classical statistical energy analysis (SEA) to the midfrequency range by establishing power balance equations between modes in different subsystems. This circumvents the requirement of the SEA approach for modal energy equipartition and enables applying SmEdA to cases of low modal over- 
lap, locally excited subsystems and complex heterogeneous subsystems. It uses the modal bases of each uncoupled subsystem. These bases can be extracted from a wider frequency range than the global modes as the extraction is performed on parts of the system considered (i.e. on subsystems). Moreover, they can be evaluated by using Finite Element models which permits dealing with subsystems having complex geometries. However, dissipative treatments have not been taken into account in the original formulation which considers constant damping loss factors for the resonant modes of each subsystem. The present study proposes to extend the method to include the dissipative effect of two types of materials widely-used in industry: viscoelastic layer patches and acoustic absorbing poroelastic materials. The methodology proposed is based on homogenized material models used to represent dissipative materials. This allows facilitating their implementation in a standard FEM code. Moreover, it reduces the computing time compared to a full model and is well-adapted for re-analysis. It is possible to calculate the normal modes related to the frequency band considered using the FE model of each subsystem, including the homogenized material model of the dissipative material. Thus the modal damping loss factors that characterize the dissipation in the SmEdA model are deduced from the equations of motions of the damped subsystems and the Galerkin method is implemented with the normal mode shapes of the subsystem considered. The modal damping loss factors are obtained from the imaginary part of the modal projection by neglecting the cross modal terms. This process can be related to the estimation of the imaginary part of the Rayleigh quotient. In the literature, it has also been called the Modal Strain Energy (MSE) method [26] in the case of a structural subsystem, and the Modal Strain and Kinetic Energy (MSKE) [27] method in the case of an acoustic subsystem. Finally, in the last step, the modal energies are obtained by resolving the SmEdA equations for each frequency band. For the purposes of validation, the extended SmEdA model proposed in this paper is applied to 
a plate-cavity test case with different configurations of dissipative treatments. The results are compared with laboratory measurements to experimentally validate the different steps of the methodology proposed. Part of this work was done during the $\mathrm{PhD}$ thesis of the first author ( [28]) and certain intermediate results were presented at two international conferences ( [28], [29], [30])

The present paper is organized as follows. The outline of SmEdA method is given in section 2 while the methodology allowing the inclusion of the dissipative material is proposed in section 3. The application to the laboratory test case is described in section 4. Finally, the experimental validations of the computed modal damping loss factors and of the subsystem energies predicted by SmEdA are presented in section 5 .

\section{Statistical modal Energy distribution Analysis Method}

Here we present the outline of the SmEdA method used to describe the power flow between different subsystems in the mid-frequency range. For the sake of clarity, this presentation focuses on a vibrating thin structure coupled to an acoustic cavity, although it can be applied to complex structures (see for instance the recent application for modelling the interaction between the floor and the interior cavity of a truck cab [31]).

In Section 2.1, we recall the results of the Dual Modal Formulation (DMF [24, 32, 33] which allows us to describe the fluid-structure interaction through the subsystem modes. Then, the SmEdA equations of motion are obtained as described in Section 2.2 by writing the power balance for each mode of each subsystem. 


\subsection{Dual modal formulation}

Let us consider the internal vibro-acoustic problem presented in Fig 1. An elastic thin panel is coupled with a rigid-walled acoustic cavity. $S$ is the fluid-structure coupling interface and $S_{r}$ is the rigid wall surface. The panel is assumed to be excited by a normal force at point $M_{e} . F_{o}(t)$ represents the force signal function of time $t$. It is assumed to be a white noise in a given frequency band of central frequency $\omega_{c}$ and of width $\Delta \omega . S_{F_{0} F_{0}}$ represents its constant power spectrum density for the frequency band considered.

A finite element discretization of the considered system is considered to simplify the presentation of the DMF. We adopt the following notation for the finite element model:

- $\mathbf{W}(t)$, the nodal displacement vector of the panel function of time;

- $\mathbf{P}(t)$, the nodal pressure vector of the cavity function of time;

- $\mathbf{F}(t)$, the nodal applied force vector on the panel due to the external mechanical excitation;

- $\mathbf{M}_{\mathrm{s}}$ and $\mathbf{K}_{\mathrm{s}}$, the mass and stiffness matrices of the finite element model of the panel, respectively;

- $\mathbf{M}_{\mathrm{c}}$ and $\mathrm{K}_{\mathrm{c}}$, the strain energy matrix and the kinetic energy matrix of the finite element model of the cavity, respectively, and;

- C, the fluid-structure coupling matrix.

The equations of motion of the conservative system can then be written [34]:

$$
\begin{gathered}
\mathbf{M}_{\mathrm{s}} \mathbf{W}^{\prime \prime}(t)+\mathbf{K}_{\mathrm{s}} \mathbf{W}(t)-\mathbf{C P}(t)=\mathbf{F}(t) \\
\mathbf{M}_{\mathrm{c}} \mathbf{P}^{\prime \prime}(t)+\mathbf{K}_{\mathrm{c}} \mathbf{P}(t)+\mathbf{C}^{\mathrm{T}} \mathbf{W}^{\prime \prime}(t)=\mathbf{0}
\end{gathered}
$$


where the superscript $\mathrm{T}$ is the matrix transpose and the apostrophe symbol refers to the derivative with time.

DMF can be used to represent the dynamic behaviour of these two coupled subsystems (i.e. vibrating structure, acoustic cavity) based on the knowledge of the uncoupled subsystem modes. This modal formulation was developed to describe the coupling between a structure and an acoustic cavity [32,33] and, more recently, it has been extended to the general case of two coupled elastic continuous mechanical systems [24].

In accordance with the DMF, the structure is described by its displacement field and uncoupled-free modes (i.e. in-vacuo modes of the structure) whereas the cavity is described by its stress fields (i.e. acoustic pressure) and uncoupled-blocked modes (i.e. rigid wall modes of the cavity). These subsystem modes can be easily calculated analytically for academic cases [32,33] or numerically with Finite Element models for complex cases [35].

In the following we use the notations below:

- $\omega_{m}, \mathbf{W}_{m}$ and $M_{m}=\mathbf{W}_{m}^{\mathrm{T}} \mathbf{M}_{\mathrm{s}} \mathbf{W}_{m}$, the angular frequency, the displacement vector and the generalized mass of mode $m$ of the in-vacuo panel;

- $\omega_{n}, \mathbf{P}_{n}$ and $M_{n}=\mathbf{P}_{n}^{\mathrm{T}} \mathbf{M}_{\mathrm{c}} \mathbf{P}_{n}$, the angular frequency, the pressure vector and the generalized 'mass' of mode $n$ of the cavity with rigid walls.

We underline that as $M_{n}$ intervenes with the second derivative with time of the modal amplitude in the modal equation, $M_{n}$ is named the modal 'mass' in the present paper as is commonly done in the literature. However, we should keep in mind that it depends on the strain energy matrix of the cavity $\mathbf{M}_{\mathrm{c}}$. 
The modal expansions of the panel displacements $\mathbf{W}$ and of the acoustic pressure $\mathbf{P}$ may be written as:

$$
\begin{aligned}
\mathbf{W}(t) & =\sum_{m \in \mathbb{N}^{*}} a_{m}(t) \mathbf{W}_{m}, \\
\mathbf{P}(t) & =\sum_{n \in \mathbb{N}^{*}} b_{n}(t) \mathbf{P}_{n},
\end{aligned}
$$

where:

- $a_{m}$ and $b_{n}$ are the amplitudes of the modes $m$ and $n$, respectively, and;

- $\mathbb{N}^{*}$ denotes the set of non-null natural numbers.

The DMF consists in: (a), introducing Eq. (34) in Eq. (1) (respectively in Eq. (2); (b), multiplying the resulting equation by $\mathbf{W}_{m}^{\mathbf{T}}$ on the left (respectively by $\mathbf{P}_{n}^{\mathbf{T}}$ ); (c), using the subsystem modal orthogonality properties $\mathbf{W}_{m^{\prime}}^{\mathbf{T}} \mathbf{M}_{\mathrm{s}} \mathbf{W}_{m}=\mathbf{W}_{m^{\prime}}^{\mathbf{T}} \mathbf{K}_{\mathrm{s}} \mathbf{W}_{m}=$ 0 for $m \neq m^{\prime}$ (respectively $\mathbf{P}_{n^{\prime}}^{\mathbf{T}} \mathbf{M}_{\mathrm{c}} \mathbf{P}_{n}=\mathbf{P}_{n^{\prime}}^{\mathbf{T}} \mathbf{K}_{\mathrm{c}} \mathbf{P}_{n}=0$ for $n \neq n^{\prime}$ ). Finally, we obtain:

$$
\begin{cases}M_{m}\left[a_{m}^{\prime \prime}(t)+\omega_{m}^{2} a_{m}(t)\right]-\sum_{n \in \mathbb{N}^{*}} W_{m n} b_{n}(t)=F_{m}(t), & \forall m \in \mathbb{N}^{*} \\ M_{n}\left[b_{n}^{\prime \prime}(t)+\omega_{n}^{2} b_{n}(t)\right]+\sum_{m \in \mathbb{N}^{*}} W_{m n} a_{m}^{\prime \prime}(t)=0, & \forall n \in \mathbb{N}^{*}\end{cases}
$$

where:

- $F_{m}=F_{0}(t) \mathbf{W}_{m}\left(M_{\mathrm{e}}\right)$ is the modal force due to the external mechanical forces, and;

- $W_{m n}$ is called the intermodal work between mode $m$ and mode $n$ and is defined by:

$$
W_{m n}=\mathbf{W}_{m}^{\mathrm{T}} \mathbf{C P}_{n}
$$


The latter quantity is related to the spatial matching of modes $m$ and $n$. It is expressed in Joules $(\mathrm{J})$.

Furthermore, if we assume that the vibratory behaviour of the coupled subsystems can be described by considering only the interaction between the resonant modes (i.e. modes in the frequency band of excitation) and if we use the change of variable $b_{n}(t)=c_{n}^{\prime}(t)$, DMF finally provides the modal equations of motion:

$$
\begin{cases}M_{m}\left[a_{m}^{\prime \prime}(t)+\omega_{m}^{2} a_{m}(t)\right]-\sum_{n \in \widetilde{N}} W_{m n} c_{n}^{\prime}(t)=F_{m}(t), & \forall m \in \widetilde{M} \\ M_{n}\left[c_{n}^{\prime \prime}(t)+\omega_{n}^{2} c_{n}(t)\right]+\sum_{m \in \widetilde{M}} W_{m n} a_{m}^{\prime}(t)=0, & \forall n \in \widetilde{N}\end{cases}
$$

where $\widetilde{M}$ and $\widetilde{N}$ are the sets of resonant modes of the structure and the cavity, respectively.

It can be observed that the system of equations (7) is interpreted as the coupling between a set of oscillators associated with the structure and another set of oscillators associated with the cavity. The coupling elements, known as gyroscopic elements, are related to the oscillators' velocities without energy dissipation (due to the opposite signs in Eq. (7p)). On the other hand, there is no direct coupling between the oscillators of the same subsystem. This mode coupling configuration is the basis of the SmEdA model. Eq. (7) has been written considering the conservative system. Terms corresponding to the modal damping models are introduced in Eq. (7) to 
take the dissipative effects into account:

$$
\begin{cases}M_{m}\left[a_{m}^{\prime \prime}(t)+\omega_{m} \eta_{m} a_{m}^{\prime}(t)+\omega_{m}^{2} a_{m}(t)\right]-\sum_{n \in \widetilde{N}} W_{m n} c_{n}^{\prime}(t)=F_{m}(t), & \forall m \in \widetilde{M} \\ M_{n}\left[c_{n}^{\prime \prime}(t)+\omega_{n} \eta_{n} c_{n}^{\prime}(t)+\omega_{n}^{2} c_{n}(t)\right]+\sum_{m \in \widetilde{M}} W_{m n} a_{m}^{\prime}(t)=0, & \forall n \in \widetilde{N}\end{cases}
$$

where $\eta_{m}$ and $\eta_{n}$ are the modal damping loss factors of the structure and the cavity, respectively. It should be underlined that we assume here that the dissipative phenomena do not introduce direct couplings between the modes of the same subsystem. This is a fundamental assumption for the development of the SEA [10] and SmEdA models [25]. However, as will be seen in section 3.3.2, cross-modal terms will appear in the modal equations of subsystems containing dissipative material. Applying SmEdA to these treated subsystems will then consist in neglecting the effect of these modal coupling terms. Hence, the SmEdA model proposed in this paper provides an approximation of the energy exchanged by the treated subsystems. Eq. (8) depends on the intermodal works $W_{m n}$ expressed by Eq. (6) as a function of the modal vectors $\mathbf{W}_{m}, \mathbf{P}_{n}$ and the coupling matrix $\mathbf{C}$. In practice, the finite element codes do not always allow the extraction of the coupling matrix. However, it has been shown (from the definition of the intermodal works for continuous systems, and using the rectangular rule for integrals) that the intermodal work can be correctly approximated using ( [24], [35]):

$$
W_{m n} \approx \sum_{i \in S} w_{m}^{i} p_{n}^{i} s^{i}
$$

where

- $w_{m}^{i}$ is the normal displacement at node $i$ of the $m^{\text {th }}$ mode;

- $p_{n}^{i}$ is the pressure at node $i$ of the $n^{\text {th }}$ mode, and; 
- $s^{i}$ is the elementary surface attributed to node $i$.

\subsection{Modal energy equations of motion}

The modal energy equations of SmEdA are obtained from the principle of energy conservation for each mode of each subsystem [25]. For mode $m$ of subsystem 1, it is written as follows:

$$
\Pi_{m}^{\mathrm{inj}}=\Pi_{m}^{\mathrm{diss}}+\sum_{n \in \widetilde{N}} \Pi_{m n}, \quad \forall m \in \widetilde{M}
$$

where:

- $\Pi_{m}^{\mathrm{inj}}$ is the time-averaged power injected by the generalized force $F_{m}$;

- $\Pi_{m}^{\text {diss }}$ is the time-averaged power dissipated by the internal damping of mode $m$;

- $\sum_{n \in \widetilde{N}} \Pi_{m n}$ is the time-averaged power flow exchanged by mode $m$ with the resonant modes of subsystem 2 .

The different powers appearing in this equation are evaluated from relations established for one single oscillator or two coupled oscillators, using the same assumptions as in SEA (white noise force spectrums, un-correlated modal interaction forces $[10,36])$ :

$$
\Pi_{m}^{\mathrm{inj}} \approx \frac{\pi}{4 M_{m}} S_{F_{0} F_{0}} w_{m}^{2}\left(M_{\mathrm{e}}\right), \quad \Pi_{m}^{\mathrm{diss}} \approx \omega_{m} \eta_{m} E_{m}, \quad \Pi_{m n} \approx \beta_{m n}\left(E_{m}-E_{n}\right)
$$

where $E_{m}$ is the time averaged energy of mode $m$ and $\beta_{m n}$ is called the intermodal coupling factor between mode $m$ and mode $n$. $\beta_{m n}$ can be decomposed as the prod- 
uct of two terms:

$$
\beta_{m n}=\beta_{m n}^{W} \times \beta_{m n}^{\omega}
$$

where

$$
\beta_{m n}^{W}=\frac{\left(W_{m n}\right)^{2}}{M_{m} M_{n}}
$$

and

$$
\beta_{m n}^{\omega}=\frac{\omega_{m} \eta_{m}\left(\omega_{n}\right)^{2}+\omega_{n} \eta_{n}\left(\omega_{m}\right)^{2}}{\left(\left(\omega_{m}\right)^{2}-\left(\omega_{m}\right)^{2}\right)^{2}+\left(\omega_{m} \eta_{m}+\omega_{n} \eta_{n}\right)\left(\omega_{m} \eta_{m}\left(\omega_{n}\right)^{2}+\omega_{n} \eta_{n}\left(\omega_{m}\right)^{2}\right)}
$$

$\beta_{m n}^{W}$ depends only on the spatial shapes of modes $m$ and $n$ (through the intermodal work). It expresses the spatial matching of the subsystem modes. On the other hand, $\beta_{m n}^{\omega}$ depends only on the natural frequencies and the damping loss factors of modes $m$ and $n$. This term expresses the frequency matching of the subsystem modes.

The power balance equation for the resonant modes of subsystem 2 can be written in the same way (taking no external source into account). Finally, we obtain a linear equation system expressing the modal energy exchanged between the two subsystems. We can write the SmEdA equations in matrix form:

$$
\left[\begin{array}{cc}
\beta_{11} & -\beta_{12} \\
-\beta_{12}^{\mathrm{T}} & \boldsymbol{\beta}_{22}
\end{array}\right]\left[\begin{array}{l}
E_{1} \\
E_{2}
\end{array}\right]=\left[\begin{array}{l}
\Pi_{1} \\
0
\end{array}\right],
$$

with $\boldsymbol{E}_{\mathbf{1}}=\left[E_{m}\right]_{\widetilde{M} \times 1}, \quad \boldsymbol{E}_{\mathbf{2}}=\left[E_{n}\right]_{\widetilde{N} \times 1}, \quad \boldsymbol{\Pi}_{\mathbf{1}}=\left[\Pi_{m}^{\mathrm{inj}}\right]_{\widetilde{M} \times 1}, \boldsymbol{\beta}_{\mathbf{1 2}}=\left[\beta_{m n}\right]_{\widetilde{M} \times \widetilde{N}}$, $\boldsymbol{\beta}_{\mathbf{1 1}}=\left[\operatorname{diag}\left(\omega_{m} \eta_{m}+\sum_{n \in \widetilde{N}} \beta_{m n}\right)\right]_{\widetilde{M} \times \widetilde{M}}, \boldsymbol{\beta}_{\mathbf{2 2}}=\left[\operatorname{diag}\left(\omega_{n} \eta_{n}+\sum_{m \in \widetilde{M}} \beta_{m n}\right)\right]_{\widetilde{N} \times \widetilde{N}}$. 
Inverting this system gives the modal energies. The overall energy of a subsystem can be obtained by summing all its modal energies and then linking the result to a spatial mean square velocity in the case of a vibrating structure, or to a mean square acoustic pressure in the case of a cavity, following standard SEA formulations [36].

These SmEdA equations depend only on the source characteristics (i.e. position, auto-spectrum density) and the modal information of each uncoupled subsystem (i.e. modal angular frequencies, modal damping loss factors, mode shapes on the coupling surface). This modal information can be obtained from finite element models for subsystems with complex geometries and/or heterogeneous mechanical properties.

We emphasize that the dissipation of energy is described in SmEdA through the modal damping loss factors. These factors do not only intervene in the modal dissipated powers, but also in the coupling strengths between the structure modes and the cavity modes (see Eq. (14) of $\beta_{m n}^{\omega}$ ). This indicates that adding a dissipative material like viscoelastic layers or a porous-elastic material in this problem will change the modal damping loss factors, and then influence the coupling strength and the power flow between the two subsystems.

Usually, for systems without dissipative treatments, the modal damping loss factors of each subsystem are assumed to be constant and equal to the estimated subsystem damping loss factors:

$$
\begin{cases}\eta_{m}=\eta^{1}, & \forall m \in \widetilde{M} \\ \eta_{n}=\eta^{2}, & \forall n \in \widetilde{N}\end{cases}
$$

where $\eta^{1}$ and $\eta^{2}$ are the damping loss factors (DLF) of the structure and the cavity, respectively. In general, these DLFs cannot be predicted for a body-in-white structure or for a non-treated cavity. They are generally estimated from measurements 
on the global system or on each uncoupled-subsystem, and by using different postprocessing techniques (Power injection method [37], ESPRIT algorithm [38, 39], etc). In the next section, we will describe a process for approximating the modal damping loss factors for subsystems containing dissipative materials.

\section{Methodology for including dissipative treatments}

In this section, a methodology for including the effect of dissipative materials in an SmEdA model is developed. Two types of dissipative materials will be considered:

- The first consists of viscoelastic layer patches, as currently used in the automotive industry for vibration control [40]. These patches are generally composed of one layer of viscoelastic material glued to one (for an unconstrained layer) or two metal sheets (for a constrained layer). Vibration energy is dissipated by the viscoelastic effect;

- The second is composed of poroelastic materials which can be used in acoustic cavities in the automotive industry for noise control. Foam and fibrous materials are typical examples of these types of materials which dissipate acoustic energy by viscous and thermal effects.

\subsection{Principle}

The methodology proposed is based on four steps:

- The first step consists in representing the dissipative materials by using homogenized material models, as described in Section 3.2.1 for the viscoelastic layer patches and in Section 3.2.2 for the poroelastic material. This type of modelling 
has two main advantages: (a) it permits increasing the upper frequency bound of the FEM calculation compared to a full detailed model; (b) it is well adapted for optimizing dissipative materials. Different positions of the dissipative materials could be considered easily by modifying only the property of the element in the original finite element mesh;

- The second step consists in building the finite element models of each subsystem treated. The dissipative treatments are introduced in these models by using the homogenized material models. As the mechanical properties of these materials may vary with frequency, constant mean values are considered for each frequency band of calculation (typically, third-octave band). A finite element model is then created for each subsystem and each frequency band. Boundary conditions at the coupling surface are defined in accordance with DMF (as discussed in section 2.1). The normal modes of each subsystem and each frequency band are then extracted. The natural frequencies and mode shapes at the coupling junction (normalized to a unit modal mass) are saved in the database used for the SmEdA calculation;

- The modal damping loss factors are estimated in the third step. Modal equations of each subsystem containing a dissipative material are obtained from the dynamic equation of motion of the subsystem considered and the Galerkin method with normal mode shapes is used. Cross-modal terms are then neglected for estimating the modal damping loss factors by analogy between the modal equations obtained and the standard ones. These developments are proposed in section 3.3 for a panel damped by viscoelastic patches and for a cavity with an absorbent material;

- The final step consists in resolving the SmEdA equations (15), depending on the subsystem modal information estimated numerically in the two previous steps. Finally, the total energy of each subsystem can be estimated by summing the 
modal energies.

\subsection{Equivalent models of dissipative materials}

In the following sub-sections, two simplified equivalent models are presented: the equivalent single layer model for describing viscoelastic layers applied to a thin structure in Section 3.2.1 and the equivalent fluid model for an acoustic cavity treated with porous materials in Section 3.2.2.

\subsubsection{Thin structure damped by viscoelastic layers}

The concept of multi-layered thin structures is increasingly used to build structures with high levels of sound and vibration performances without significant addition of weight. However, modelling such structures is not an easy task. Hence it is possible to consider a 3-D finite element model representing the viscoelastic layers coupled to a 2D finite element model representing the thin structures [41]. However, attention must be given to the sizes of the elements to accurately represent the behaviour of the multilayer structure. The number of degrees of freedom can become significant. Moreover, this specific model of the viscoelastic layer patches requires re-meshing the system if the patches are moved in view to optimizing vibration and noise. Another strategy adopted in this paper and illustrated in Fig. 2 consists in evaluating the single layer properties equivalent to the multi-layered structures [40]. It permits dealing with any number of layers and facilitates the modification of the material properties of each layer. It also facilitates the positioning of the viscoelastic patches on the structure by modifying the element properties of the 2D shell finite element model of the structure.

The homogenization method clearly described in [42,43] is based on a travelling 
wave approach where continuity conditions on displacement and shear stresses at each layer interface are used to obtain the equations of motion of the multi-layered plate field expressed in the function of the first layer field. Without entering into detail, the method estimates the material properties of the equivalent one single layer structure as a function of the material parameters and thickness of each layer, that is: $E_{\mathrm{eq}, \mathrm{m}}^{*}$, the homogenized complex Young's modulus; $v_{\mathrm{eq}, \mathrm{m}}=\frac{\sum_{i} h_{i} v_{i}}{\sum_{i} h_{i}}$, the equivalent Poisson's ratio; and $\rho_{\text {eq,m }}$, the equivalent homogenized density. The subscripts "eq, m" and "i" refer to the equivalent homogenized parameters of the multilayer and $i^{\text {th }}$ layer, respectively. The equivalent homogenized damping loss factor $\eta_{\mathrm{eq}, \mathrm{m}}$ is then given by:

$$
\eta_{\text {eq, } \mathrm{m}}=\frac{\operatorname{Im}\left\{E_{\mathrm{eq}, \mathrm{m}}^{*}\right\}}{\operatorname{Re}\left\{E_{\mathrm{eq}, \mathrm{m}}^{*}\right\}}
$$

It must be emphasized that the resulting equivalent Young's modulus and damping loss factor may depend on the frequency. In the following description of the methodology proposed, averaged values for each third octave band will be considered for the frequency-dependant physical parameters. This is applied in particular to the equivalent Young's modulus and the damping loss factors. The approach described in this section was programmed using an in-house Fortran code called MOVISAND and validated numerically by comparison with 3D - FEM calculations [44].

\subsubsection{Acoustic cavity including poroelastic materials}

Biot's theory [45] is commonly used when modelling poroelastic materials like fibrous materials or various types of foams. However, it requires knowledge of a large number of physical parameters that are not all easily measurable. To overcome this difficulty, the equivalent fluid model $[8,46-48]$ is often used as an alternative 
since it considerably simplifies the Biot model. In some situations, the effect of the solid phase deformation on the material response can be neglected and the problem can be formulated in terms of fluid pressure only. This is verified when the fluid inertial forces dominate the elastic ones or when the elastic stresses are weak [47]. The first case (i.e. rigid model, [49]) can be found when the porous material is placed in front of the vibrating panel without being coupled to it whereas the second one (i.e. limp model, [50]) is found when the bulk modulus of the porous skeleton is close to zero (for instance, for glass-wool or fibrous materials). As light weight fibrous materials are generally used for noise control in car and truck cabins, the limp model might be more pertinent than the rigid model for our applications. In the following, we consider the equivalent fluid model which describes the behaviour of the fluid phase with an equation similar to the Helmholtz one:

$$
\frac{\Delta P}{\rho_{\mathrm{eq}, \mathrm{f}}^{*}}-\omega^{2} \frac{P}{K_{\mathrm{eq}, \mathrm{f}}^{*}}=0
$$

where $\rho_{\text {eq,f }}^{*}$ and $K_{\text {eq,f }}^{*}$ are the complex equivalent fluid density and the complex equivalent fluid compressibility, respectively.

Whereas the Biot model requires at least five different macroscopic material parameters, the equivalent fluid is characterized by only two parameters (i.e. $\rho_{\mathrm{eq}, \mathrm{f}}^{*}$ and $K_{\text {eq,f }}^{*}$ ). The latter can be estimated from acoustic tube measurements. The two cavities method proposed by Utsuno et al. [51] gives the characteristic impedance $Z_{\mathrm{c}}^{*}$ and the propagation constant $k_{\text {eq,f }}^{*}$ of the material from transfer functions measured with two different back cavities behind the material. The equivalent fluid parameters are deduced by:

$$
K_{\mathrm{eq}, \mathrm{f}}^{*}=\frac{Z_{\mathrm{c}}^{*} \omega}{k_{\mathrm{eq}, \mathrm{f}}^{*}}
$$


and

$$
\rho_{\mathrm{eq}, \mathrm{f}}^{*}=\frac{\left(Z_{\mathrm{c}}^{*}\right)^{2}}{K_{\mathrm{eq}, \mathrm{f}}^{*}}
$$

These two quantities are complex numbers. Therefore, we can express two damping loss factors:

$$
\chi_{\mathrm{eq}, \mathrm{f}}=\frac{\operatorname{Im}\left\{\rho_{\mathrm{eq}, \mathrm{f}}^{*}\right\}}{\operatorname{Re}\left\{\rho_{\mathrm{eq}, \mathrm{f}}^{*}\right\}}
$$

and

$$
\eta_{\text {eq, } \mathrm{f}}=\frac{\operatorname{Im}\left\{K_{\mathrm{eq}, \mathrm{f}}^{*}\right\}}{\operatorname{Re}\left\{K_{\mathrm{eq}, \mathrm{f}}^{*}\right\}}
$$

$\chi_{\text {eq, }}$ is a negative quantity related to the inertial characteristic of the equivalent fluid and expresses the dissipation of energy by viscous effect [52]. On the other hand, $\eta_{\text {eq,f }}$ is a positive quantity related to the compressibility characteristic of the equivalent fluid and expresses the dissipation of energy by thermal effect [52]. In addition, a constant density for the air $\rho_{\text {air }}$ and a constant real part of the fluid compressibility $K_{\text {air }}^{*}$ will be considered in the following, whereas the damping loss factor of the air $\eta_{\text {air }}=\frac{\operatorname{Im}\left\{K_{\text {air }}^{*}\right\}}{\operatorname{Re}\left\{K_{\text {air }}^{*}\right\}}$ will be estimated experimentally for the empty cavity.

\subsection{Estimation of the modal damping loss factor}

\subsubsection{Finite element model}

For each subsystem treated (i.e. structure, cavity), and for each frequency band, a finite element (FE) model is created including the homogenized model of the dissipative material.

\section{- Plate damped by viscoelastic patches}


The damped structure is meshed by 2D shell elements. The properties obtained with the homogenized material model described in section 3.2 .1 are assigned to the elements corresponding to the patch positions, whereas the mechanical properties of the other elements of the mesh correspond to the untreated structure.

The equations of motion of the FE model of the structure treated can be written in the frequency domain:

$$
\left\{\mathbf{K}_{\mathrm{ba}}^{*}+\mathbf{K}_{\mathrm{eq}, \mathrm{m}}^{*}-\omega^{2}\left(\mathbf{M}_{\mathrm{ba}}+\mathbf{M}_{\mathrm{eq}, \mathrm{m}}\right)\right\} \mathbf{W}=\mathbf{F}
$$

where the subscripts "ba" and "eq,m" refer to the bared part and the equivalent multilayer part of the structure, respectively. $\mathbf{K}_{\mathrm{ba}}^{*}$ and $\mathbf{K}_{\mathrm{eq}, \mathrm{m}}^{*}$ are the complex stiffness matrices whereas $\mathbf{M}_{\mathrm{ba}}$ and $\mathbf{M}_{\mathrm{eq}, \mathrm{m}}$ are the mass matrices.

Taking into account that each part is characterized by a complex Young's modulus (i.e. $E_{\mathrm{ba}}^{*}$ and $E_{\mathrm{eq}, \mathrm{m}}^{*}$ ), the complex stiffness matrices can be written as:

$$
\begin{aligned}
& \mathbf{K}_{\mathrm{ba}}^{*}=E_{\mathrm{ba}}^{*} \mathbf{K}_{\mathrm{ba}}^{\mathrm{e}}=\left(1+j \eta_{\mathrm{ba}}\right) \overline{\mathbf{K}}_{\mathrm{ba}} \\
& \mathbf{K}_{\mathrm{eq}, \mathrm{m}}^{*}=E_{\mathrm{eq}, \mathrm{m}}^{*} \mathbf{K}_{\mathrm{eq}, \mathrm{m}}^{\mathrm{e}}=\left(1+j \eta_{\mathrm{eq}, \mathrm{m}}\right) \overline{\mathbf{K}}_{\mathrm{eq}, \mathrm{m}}
\end{aligned}
$$

where $\mathbf{K}_{\mathrm{ba}}^{\mathrm{e}}$ and $\mathbf{K}_{\mathrm{eq}, \mathrm{m}}^{\mathrm{e}}$ are the stiffness elementary matrices, $\overline{\mathbf{K}}_{\mathrm{ba}}$ and $\overline{\mathbf{K}}_{\mathrm{eq}, \mathrm{m}}$ are the stiffness matrices of the conservative (i.e. undamped) system.

\section{- Cavity with an absorbent material}

For the damped cavity, the cavity and the porous materials are meshed by $3 \mathrm{D}$ elements. The properties obtained with the equivalent fluid model described in section 3.2.2 are assigned to the poroelastic material elements, while the air properties are assigned to the air elements. 
The equations of motion of the FE model of the cavity treated can be written in the frequency domain [8]:

$$
\left\{\mathbf{K}_{\mathrm{air}}+\mathbf{K}_{\mathrm{eq}, \mathrm{f}}^{*}-\omega^{2}\left(\mathbf{M}_{\mathrm{air}}^{*}+\mathbf{M}_{\mathrm{eq}, \mathrm{f}}^{*}\right)\right\} \mathbf{P}=\omega^{2} \mathbf{C}^{\mathrm{T}} \mathbf{W}
$$

The subscripts "air" and "eq,f" refer to the air and the equivalent fluid, respectively. $\mathbf{K}_{\text {air }}$ and $\mathbf{K}_{\mathrm{eq}, \mathrm{f}}^{*}$ are the kinetic energy matrices, $\mathbf{M}_{\text {air }}^{*}$ and $\mathbf{M}_{\mathrm{eq}, \mathrm{f}}^{*}$ are the strain energy matrices.

Taking into account that each part is described by a density $\left(\rho_{\text {air }}, \rho_{\text {eq, }}^{*}\right)$ and a fluid compressibility $\left(K_{\text {air }}^{*}, K_{\text {eq,f }}^{*}\right.$ ) and assuming that $\eta_{\text {air }}<<1, \eta_{\text {eq,f }}<<1$, the following equations can be written as:

$$
\begin{aligned}
& \mathbf{K}_{\text {air }}=\frac{1}{\rho_{\text {air }}} \mathbf{K}_{\text {air }}^{\mathrm{e}}=\overline{\mathbf{K}}_{\mathrm{air}} \\
& \mathbf{M}_{\mathrm{air}}^{*}=\frac{1}{K_{\mathrm{air}}^{*}} \mathbf{M}_{\mathrm{air}}^{\mathrm{e}}=\left(1-j \eta_{\mathrm{air}}\right) \overline{\mathbf{M}}_{\mathrm{air}} \\
& \mathbf{K}_{\mathrm{eq}, \mathrm{f}}^{*}=\frac{1}{\rho_{\mathrm{eq}, \mathrm{f}}^{*}} \mathbf{K}_{\mathrm{eq}, \mathrm{f}}^{\mathrm{e}}=\frac{1-j \chi_{\mathrm{eq}, \mathrm{f}}}{1+\chi_{\mathrm{eq}, \mathrm{f}}^{2}} \overline{\mathbf{K}}_{\mathrm{eq}, \mathrm{f}} \\
& \mathbf{M}_{\mathrm{eq}, \mathrm{f}}^{*}=\frac{1}{K_{\mathrm{eq}, \mathrm{f}}^{*}} \mathbf{M}_{\mathrm{eq}, \mathrm{f}}^{\mathrm{e}}=\left(1-j \eta_{\mathrm{eq}, \mathrm{f}}\right) \overline{\mathbf{M}}_{\mathrm{eq}, \mathrm{f}}
\end{aligned}
$$

where the matrices with the superscript "e" are the elementary matrices and the matrices with the bar symbol " - " are the matrices of the conservative system.

The estimation of the modal loss factors for each subsystem is proposed in the next section from the FE equations of motion.

\subsubsection{Galerkin method considering the normal subsystem modes}

\section{- Plate damped by viscoelastic patches}


Let us consider the normal mode $m$ satisfying the eigenvalue problem of the conservative system:

$$
\left\{\overline{\mathbf{K}}_{\mathrm{ba}}+\overline{\mathbf{K}}_{\mathrm{eq}, \mathrm{m}}-\omega_{m}^{2}\left(\mathbf{M}_{\mathrm{ba}}+\mathbf{M}_{\mathrm{eq}, \mathrm{m}}\right)\right\} \mathbf{W}_{m}=0
$$

The Galerkin method consists in introducing the modal expansion of the displacement (3) in Eq. 23) and in multiplying the resulting equation by $\mathbf{W}_{m}^{\mathrm{T}}$ on the left. By taking into account the subsystem mode orthogonality properties (i.e. $\mathbf{W}_{m^{\prime}}^{\mathrm{T}}\left(\mathbf{M}_{\mathrm{ba}}+\right.$ $\left.\mathbf{M}_{\mathrm{eq}, \mathrm{m}}\right) \mathbf{W}_{m}=0, \mathbf{W}_{m^{\prime}}^{\mathrm{T}}\left(\mathbf{K}_{\mathrm{ba}}+\mathbf{K}_{\mathrm{eq}, \mathrm{m}}\right) \mathbf{W}_{m}=0$ for $\left.m^{\prime} \neq m\right)$, we obtain the modal equation:

$\left(\omega_{m}^{2}-\omega^{2}\right) M_{m} a_{m}+j \sum_{m^{\prime} \in \mathbb{N}^{*}}\left[\eta_{\mathrm{ba}} \mathbf{W}_{m}^{\mathrm{T}} \overline{\mathbf{K}}_{\mathrm{ba}} \mathbf{W}_{m^{\prime}}+\eta_{\mathrm{eq}, \mathrm{m}} \mathbf{W}_{m}^{\mathrm{T}} \overline{\mathbf{K}}_{\mathrm{eq}, \mathrm{m}} \mathbf{W}_{m^{\prime}}\right] a_{m^{\prime}}=\mathbf{W}_{m}^{\mathrm{T}} \mathbf{F}$

These equations contain cross-modal terms (i.e. $m^{\prime} \neq m$ ). This is due to the fact that the normal modes of the conservative system have been considered instead of the complex modes of the non-conservative system. It is well-known that in some situations (for instance when the viscoelastic layer covers a large part of the panel considered and the damping loss factors are small compared to one), these cross-modal terms can be neglected. This is generally called the Rayleigh or Basile assumption [53]. On the contrary, for systems with locally highly damped material, they may play a significant role in describing the vibratory field in the vicinity of the damped material. In the framework of the methodology proposed, we neglect these cross modal terms. Consequently, the results of our approach will be an approximation of the global energy of each subsystem. By neglecting these terms, Eq. (28) can be approximated by:

$$
\left[\omega_{m}^{2}\left\{1+j \frac{\eta_{\mathrm{ba}} \mathbf{W}_{m}^{\mathrm{T}} \overline{\mathbf{K}}_{\mathrm{ba}} \mathbf{W}_{m}+\eta_{\mathrm{eq}, \mathrm{m}} \mathbf{W}_{m}^{\mathrm{T}} \overline{\mathbf{K}}_{\mathrm{eq}, \mathrm{m}} \mathbf{W}_{m}}{\omega_{m}^{2} M_{m}}\right\}-\omega^{2}\right] M_{m} a_{m} \approx \mathbf{W}_{m}^{\mathrm{T}} \mathbf{F}
$$


By analogy with the standard modal equation of motion $\left[\omega_{m}^{2}\left\{1+j \eta_{m}\right\}-\omega^{2}\right] M_{m} a_{m}=$ $\mathbf{W}_{m}^{T} \mathbf{F}$ where $\eta_{m}$ is the modal damping loss factor, we identify:

$$
\eta_{m} \approx \frac{\eta_{\mathrm{ba}} \mathbf{W}_{m}^{\mathrm{T}} \overline{\mathbf{K}}_{\mathrm{ba}} \mathbf{W}_{m}+\eta_{\mathrm{eq}, \mathrm{m}} \mathbf{W}_{m}^{\mathrm{T}} \overline{\mathbf{K}}_{\mathrm{eq}, \mathrm{m}} \mathbf{W}_{m}}{\omega_{m}^{2} M_{m}}
$$

\section{- Cavity with an absorbent material}

Let us consider that the normal mode $n$ satisfies the eigenvalue problem of the conservative system:

$$
\left\{\overline{\mathbf{K}}_{\text {air }}+\overline{\mathbf{K}}_{\text {eq,f }}-\omega_{n}^{2}\left(\overline{\mathbf{M}}_{\text {air }}+\overline{\mathbf{M}}_{\text {eq,f }}\right)\right\} \mathbf{P}_{n}=0
$$

The Galerkin method is applied again by introducing the modal expansion (4) in Eq. 25 and by multiplying the resulting equation by $\mathbf{P}_{n}^{\mathrm{T}}$ on the left. The mode orthogonality properties are considered (i.e. $\mathbf{P}_{n^{\prime}}^{\mathrm{T}}\left(\overline{\mathbf{M}}_{\mathrm{air}}+\overline{\mathbf{M}}_{\mathrm{eq}, \mathrm{f}}\right) \mathbf{P}_{n}=0, \mathbf{P}_{n^{\prime}}^{\mathrm{T}}\left(\overline{\mathbf{K}}_{\mathrm{air}}+\right.$ $\left.\overline{\mathbf{K}}_{\text {eq, } \mathrm{f}}\right) \mathbf{P}_{n}=0$ for $n^{\prime} \neq n$ ), but certain cross modal terms (i.e. $\mathbf{P}_{n}^{\mathrm{T}} \overline{\mathbf{K}}_{\mathrm{eq}, \mathrm{f}} \mathbf{P}_{n^{\prime}}$, $\mathbf{P}_{n}^{\mathrm{T}} \overline{\mathbf{M}}_{\mathrm{air}} \mathbf{P}_{n^{\prime}}, \mathbf{P}_{n}^{\mathrm{T}} \overline{\mathbf{M}}_{\mathrm{eq}, \mathrm{f}} \mathbf{P}_{n^{\prime}}$ for $n^{\prime} \neq n$ ) do not vanish. As with the damped panel, we are led to neglect these cross-modal terms. We then obtain:

$$
\begin{aligned}
{\left[\bar{\omega}_{n}^{2}\left\{1-j \frac{\chi_{\text {eq, } \mathrm{f}}}{1+\chi_{\text {eq }, \mathrm{f}}^{2}} \frac{\mathbf{P}_{n}^{\mathrm{T}} \overline{\mathbf{K}}_{\text {eq,f }} \mathbf{P}_{n}}{\bar{\omega}_{n}^{2} M_{n}}\right\}\right.} & -\omega^{2}\left\{1-j \frac{1}{M_{n}}\left(\eta_{\text {air }} \mathbf{P}_{n}^{\mathrm{T}} \overline{\mathbf{M}}_{\text {air }} \mathbf{P}_{n}\right.\right. \\
& \left.\left.\left.+\eta_{\text {eq }, \mathrm{f}} \mathbf{P}_{n}^{\mathrm{T}} \overline{\mathbf{M}}_{\text {eq }, \mathrm{f}} \mathbf{P}_{n}\right)\right\}\right] M_{n} b_{n} \approx \omega^{2} \mathbf{P}_{n}^{\mathrm{T}} \mathbf{C}^{\mathrm{T}} \mathbf{W}
\end{aligned}
$$

where $\bar{\omega}_{n}^{2}$ is the modified eigenfrequency induced by the viscous effect of the poroelastic material:

$$
\bar{\omega}_{n}^{2}=\frac{1}{M_{n}}\left(\mathbf{P}_{n}^{\mathrm{T}} \overline{\mathbf{K}}_{\mathrm{air}} \mathbf{P}_{n}+\frac{1}{1+\chi_{\mathrm{eq}, \mathrm{f}}^{2}} \mathbf{P}_{n}^{\mathrm{T}} \overline{\mathbf{K}}_{\mathrm{eq}, \mathrm{f}} \mathbf{P}_{n}\right)
$$


By assuming that the modal damping effects are essentially produced at the resonance frequency $\left(\omega=\bar{\omega}_{n}\right)$, Eq. (32) becomes:

$$
\begin{array}{r}
{\left[\bar{\omega}_{n}^{2}\left\{1+j \frac{1}{M_{n}}\left(-\frac{\chi_{\mathrm{eq}, \mathrm{f}}}{1+\chi_{\mathrm{eq}, \mathrm{f}}^{2}} \frac{\mathbf{P}_{n}^{\mathrm{T}} \overline{\mathbf{K}}_{\mathrm{eq}, \mathrm{f}} \mathbf{P}_{n}}{\bar{\omega}_{n}^{2}}+\eta_{\mathrm{air}} \mathbf{P}_{n}^{\mathrm{T}} \overline{\mathbf{M}}_{\mathrm{air}} \mathbf{P}_{n}+\eta_{\mathrm{eq}, \mathrm{f}} \mathbf{P}_{n}^{\mathrm{T}} \overline{\mathbf{M}}_{\mathrm{eq}, \mathrm{f}} \mathbf{P}_{n}\right)\right\}\right.} \\
\left.-\omega^{2}\right] b_{n} \approx \omega^{2} \mathbf{P}_{n}^{\mathrm{T}} \mathbf{C}^{\mathrm{T}} \mathbf{W}
\end{array}
$$

Finally, the modal damping loss factor can be deduced through comparison with the standard modal equation:

$$
\eta_{n} \approx \frac{1}{M_{n}}\left(-\frac{\chi_{\mathrm{eq}, \mathrm{f}}}{1+\chi_{\mathrm{eq}, \mathrm{f}}^{2}} \frac{\mathbf{P}_{n}^{\mathrm{T}} \overline{\mathbf{K}}_{\mathrm{eq}, \mathrm{f}} \mathbf{P}_{n}}{\bar{\omega}_{n}^{2}}+\eta_{\mathrm{air}} \mathbf{P}_{n}^{\mathrm{T}} \overline{\mathbf{M}}_{\mathrm{air}} \mathbf{P}_{n}+\eta_{\mathrm{eq}, \mathrm{f}} \mathbf{P}_{n}^{\mathrm{T}} \overline{\mathbf{M}}_{\mathrm{eq}, \mathrm{f}} \mathbf{P}_{n}\right)
$$

In conclusion, we use Eq. (30) and (35) to estimate the modal damping loss factors of the panel damped by viscoelastic patches, and of the cavity including absorbing material, respectively. These formula have been obtained by neglecting cross-modal terms resulting from the modal projection. Comparisons between these off-diagonal terms with the diagonal terms of the modal damping matrix will be presented in section 4.4 in the test case. 


\section{Test Case Application}

\subsection{Description of the test case and the different configurations of dissipative materials}

\subsubsection{Plate-Cavity system}

The cavity-panel system described in Fig. 3 is considered for the present application. The vibrating rectangular panel is coupled with a rigid-walled rectangular acoustic cavity. The homogeneous plate is made of steel (mass density $\rho=$ $7800 \mathrm{~kg} \mathrm{~m}^{-3}$, with a Young's modulus of $E=2 \times 10^{11} \mathrm{~Pa}$ ) and its dimensions are $0.5 \mathrm{~m} \times 0.6 \mathrm{~m} \times 1 \mathrm{~mm}$. It is assumed to be clamped at its 4 edges and excited by a normal point force exerted at point $M_{\mathrm{e}}$ as shown in Fig. 3. The cavity is filled with air (mass density $\rho_{\text {air }}=1.29 \mathrm{~kg} \mathrm{~m}^{-3}$, celerity $c_{\text {air }}=342 \mathrm{~m} \mathrm{~s}^{-1}$ ) and has a depth of $0.7 \mathrm{~m}$ (z-axis). In terms of modelling, the behaviour of the plate is described by the Kirchhoff equation, whereas the Helmholtz equation is considered for the fluid domain. In the following, this configuration without any dissipative treatment will be called the 'Bare' configuration.

\subsubsection{Configurations of dissipative materials}

In addition to the 'Bare' configuration, three configurations with dissipative treatments applied to the plate-cavity system are considered, as shown in Fig. 4 . They are named and defined as follows:

- Viscl configuration: The plate is treated with one viscoelastic damping patch whereas the cavity remains empty.

- Visc2 configuration: The plate is treated with two viscoelastic damping patches 
whereas the cavity remains empty.

- Poro configuration: The cavity is treated with a fibrous material whereas the plate remains untreated.

The viscoelastic layer is a $2.6 \mathrm{~mm}$ thick prefabricated damping patch currently used in the automotive industry. It can be semi-permanently attached to the plate by heating. A single damping patch occupies $15 \%$ of the plate surface area and its mass is approximately $19 \%$ of the plate mass.

The fibrous material consists of $3 \mathrm{~cm}$ thick mineral fiber fixed at the bottom of the cavity. It occupies around $2 \%$ of the cavity volume.

\subsection{Equivalent parameters of the dissipative treatments}

\subsubsection{Plate with the viscoelastic patch}

The mechanical properties of the homogenized material model of the viscoelastic layer patch (i.e. steel plate + viscoelastic layer) are estimated as described in section 3.2.1 using the equivalent single layer model implemented with MOVISAND software. The complex elastic modulus of the viscoelastic material $E_{\text {visc }}^{*}$ is determined using the time-temperature superposition principle (empirical WilliamLandel-Ferry "WLF" equation) [54]. The frequency-dependent curves of isothermal properties at different temperatures were estimated experimentally by the manufacturer using a dynamic mechanical analyser (DMA+300 from METRAVIB company). Measurements were performed at several temperatures from $-30{ }^{\circ} \mathrm{C}$ to $70{ }^{\circ} \mathrm{C}$ (step of $10^{\circ} \mathrm{C}$ ) in a frequency range from 1 to $200 \mathrm{~Hz}$, giving a reliable Young's modulus and loss factor extraction at $20^{\circ} \mathrm{C}$ for the whole audible range.

Table 1 and Fig. 5 present the mechanical properties of the plate steel, the vis- 
coelastic material at $20{ }^{\circ} \mathrm{C}$ and the equivalent model estimated with MOVISAND. Note that the damping loss factor of the plate steel was estimated experimentally on the bare plate using the high-resolution modal analysis method [39], as described in Appendix A. It can be observed that the equivalent parameters $\left(E_{e q}^{*}, \eta_{e q}\right)$ vary gradually with frequency. Although these quantities are frequency-dependent, the slight variations make it reasonable to consider averaged values for each third octave and to perform modal analysis for each of these bands.

\subsubsection{Cavity with the fibrous material}

The equivalent characteristic impedance $Z_{c}^{*}$ and the equivalent wavenumber $k_{e q}^{*}$ of the fibrous material were estimated with two impedance tubes ( $B \& K$ type 4206$)$. A large $10 \mathrm{~cm}$ diameter tube was used for frequencies up to $1.6 \mathrm{kHz}$ whereas a small $3 \mathrm{~cm}$ diameter tube was used from $1.6 \mathrm{kHz}$ to $6.4 \mathrm{kHz}$. The equivalent fluid parameters of the fibrous material are then deduced from Eq. (19) and Eq. (20). The values averaged for each third octave band are shown in Fig. 6, The equivalent density decreases as a function of the frequency whereas the equivalent compressibility increases. These results are in accordance with the Delany-Bazelay model [55] considering the flow resistivity of the fibrous material. It can be seen that the $\chi_{\text {eq,f }}$ damping loss factors are significant compared to unity, justifying it being taken into account (compared to one) in Eq. 26 .

\subsection{Modal analysis of subsystems}

Once the parameters of the equivalent models for the viscoelastic multilayer and the fibrous material were estimated, a finite element mesh of each subsystem was created. The plate was represented with 13776 quadrilateral shell elements whereas 
the cavity was represented with 4031412 tetrahedral solid elements. The mesh sizes were chosen in order to have a minimum of six elements per bending wavelength at $8 \mathrm{kHz}$ for the plate and per acoustic wavelength at $6 \mathrm{kHz}$ for the cavity. Then, we checked that these element sizes permitted conforming to the criterion of six elements per wavelength for the equivalent single layer model and for the equivalent fluid for all the frequencies between $315 \mathrm{~Hz}$ and $8 \mathrm{kHz}$ for the plate and between $315 \mathrm{~Hz}$ and $6 \mathrm{kHz}$ for the cavity. It should be underlined that the validity of the cavity mesh was limited to $6 \mathrm{kHz}$ (compared to $8 \mathrm{kHz}$ for the plate) because no fibrous properties above this frequency were available. For the treated configurations, the properties of the elements corresponding to the dissipative material were based on the properties of the equivalent model for the third octave band considered. For the other mesh elements, their properties corresponded to those described in section 4.1.1 for the bare case. The damping loss factors were estimated experimentally, as described in section 4.2.1 for the steel plate and as will be described in section 4.4 .2 for the air cavity.

The modal analysis was performed for each subsystem for each third octave band using the MSC/NASTRAN code and the Lanczos method. A NASTRAN DMAP program allowed estimating the modal damping loss factors (see Eq. (30) and Eq. (35)) in the same run as the modal analysis. The natural frequencies, the mode shapes normalized to a unit modal mass (i.e. $M_{m}=1 \mathrm{~kg} \mathrm{~m}^{2}$ for a panel mode $m$ or $M_{n}=1 \mathrm{~kg} \mathrm{~m}^{2} \mathrm{~s}^{-2}$ for a cavity mode $n$ ), and the damping loss factors for all the modes of the frequency band considered were saved in a MATLAB file at the end of the calculation. The process was automated as it was repeated for each frequency band of interest. For the different frequency bands, a MATLAB program allowed creating the input NASTRAN data file with the appropriate properties for the elements corresponding to the equivalent material (depending on the frequency band), in order to run the NASTRAN calculation and export the NASTRAN outputs in a 
MATLAB file.

Table 2 presents the number of subsystem modes per third octave band for each configuration. It can be seen that in general the configurations treated have only slightly fewer modes than the bare case, indicating that the modal densities are not greatly affected by the considered dissipative materials.

\subsection{Modal damping loss factor results}

\subsubsection{Plate with the viscoelastic patches}

Eq. (29) was obtained from Eq (28) by neglecting the off-diagonal terms of the modal damping matrix (in comparison with the diagonal terms). To illustrate this assumption in the present case, Fig. 7 7 shows the modal damping matrix for the first ten modes of the third octave band $1600 \mathrm{~Hz}$. It can be seen that the assumption is correctly respected for the Visc2 plate whereas several off-diagonal terms presents moderate values for several couples of modes for the Viscl plate. For latter case, it can be seen that the mode presenting the lower value on the diagonal matrix (i.e. $n=7$ ) is weakly coupled with the other modes. For all the other modes, the term on the diagonal also dominates the off-diagonal terms. The assumption is then globally verified for these modes of the Viscl plate. This figure clearly highlights that the validity of the assumption depends on the distribution of the viscoelastic patches. The best case would be when the viscoelastic layer covers entirely the plate. The modal damping matrix would then be diagonal (resulting from the orthogonality property of the modeshapes).

Fig. 8 presents the modal damping loss factors of Visc1 and Visc2 plates in comparison with the damping loss factors of the steel plate and the equivalent model of the 
viscoelastic multilayer. It can be seen that the damping pads provide substantially high damping compared to the bare plate. Their effects decrease slightly with increasing frequencies, similar to the decreasing values of the damping loss factors of the equivalent viscoelastic model. The damping values can vary substantially from one mode to another for the same configuration. This indicates that some modes exhibiting low strains at the pad positions are less influenced by the damping pad than others. This is illustrated in Fig. 9, where the spatial shapes of two modes of Visc1 (identified by two red circles on Fig. 8) are plotted: the first has a natural frequency of $1584 \mathrm{~Hz}$ and a damping loss factor of 0.007 , whereas the second has a natural frequency of $1818 \mathrm{~Hz}$ and a damping loss factor of 0.05 . It can be clearly seen that the first mode does not exhibit significant spatial deformation around the damping pad, contrary to the second mode. Compared to the Viscl configuration, Visc2 presents averaged values roughly 50\% higher and lower variations from one mode to another. This can be explained by the fact that the two damping pads occupied an area twice as large as a single pad.

\subsubsection{Cavity with fibrous material}

To study the cross-modal terms neglected in Eq. (32), two modal matrices present in the Galerkin method are plotted in Fig 10 . These results concern the first ten cavity modes of the third octave band $1000 \mathrm{~Hz}$. In Fig. 10a, the modal matrix concerns the air cavity. The off-diagonal terms are negligible compared to the diagonal ones. This result is not surprising because the air cavity occupies almost the whole volume of the subsystem while the pressure modeshapes satisfy its property of orthogonality. On the contrary, several off-diagonal terms are significant in Fig. $10 \mathrm{~b}$ which concerns the equivalent fluid domain (i.e. fibrous material). This results from the fact that the fibrous material occupies only a small volume of the subsystem. 
However, for a given mode $n$, the diagonal term is generally greater than the offdiagonal terms concerning the same mode. This may lead to the assumption that the coupling between the different modes (having different natural frequencies) is weak. Modes $n=4$ and $n=9$ are exceptions to this rule as the off-diagonal term is equivalent to the diagonal one. As with the damped panel, this clearly underlines that the assumption of neglecting the cross-modal terms in the Galerkin method is a considerable approximation in the estimation of the modal damping loss factors.

The damping loss factors of the cavity treated with the fibrous material were estimated with Eq. (35) and plotted in Fig. 11. The damping loss factors of the empty cavity are also shown in this figure for comparison. These values were estimated experimentally when the cavity was closed with a $12 \mathrm{~cm}$ thick concrete panel. As for the bare plate, the high-resolution modal analysis method [39] was used to estimate the modal damping of resonant modes. We underline that these values were considered in the FE model for the elements corresponding to the non-treated part of the air cavity.

Fig. 11a presents the contributions of the different parts of Eq. 35):

- $\eta_{\mathrm{MKE}}=-\frac{\chi_{\mathrm{eq}, \mathrm{f}}}{1+\chi_{\mathrm{eq}, \mathrm{f}}^{2}} \frac{\mathbf{P}_{n}^{\mathrm{T}} \overline{\mathbf{K}}_{\mathrm{eq}, \mathrm{f}} \mathbf{P}_{n}}{\bar{\omega}_{n}^{2} M_{n}}$ depending on the modal kinetic energy [27] of the equivalent fluid (i.e. $\mathbf{P}_{n}^{\mathrm{T}} \overline{\mathbf{K}}_{\text {eq,f }} \mathbf{P}_{n}$ ), and,

- $\eta_{\mathrm{MSE}}=\frac{1}{M_{n}}\left[\eta_{\mathrm{air}}\left(\mathbf{P}_{n}^{\mathrm{T}} \overline{\mathbf{M}}_{\mathrm{air}} \mathbf{P}_{n}\right)+\eta_{\mathrm{eq}, \mathrm{f}}\left(\mathbf{P}_{n}^{\mathrm{T}} \overline{\mathbf{M}}_{\mathrm{eq}, \mathrm{f}} \mathbf{P}_{n}\right)\right]$ depending on the modal strain energies [26] of the air cavity (i.e. $\mathbf{P}_{n}^{\mathrm{T}} \overline{\mathbf{M}}_{\text {air }} \mathbf{P}_{n}$ ) and of the equivalent fluid (i.e. $\left.\mathbf{P}_{n}^{\mathrm{T}} \overline{\mathbf{M}}_{\mathrm{eq}, \mathrm{f}} \mathbf{P}_{n}\right)$.

It can be seen that the first terms are in general predominant, except below $500 \mathrm{~Hz}$ and for some high frequency modes. This indicates that the viscous effect leads to more energy dissipation than the thermal effect. It should be emphasized here that the modal damping factors are not directly proportional to the sound pressure. This 
would lead to erroneous modelling of the viscous effect, since the normal fluid particle velocity close to a rigid wall is low whereas the acoustic pressure is generally significant. In the present approach, the viscous effect is represented by the term $\eta_{\mathrm{MKE}}$, which is proportional to the modal kinetic energy of the equivalent fluid. This depends on the pressure gradient, and then on the acoustic velocity. This particular point is illustrated on the mode of natural frequency $469 \mathrm{~Hz}$. The pressure modeshape presents significant values at the location of the absorbing material, as shown in Fig. 12a whereas $\eta_{\text {MKE }}$ is small compared to $\eta_{\text {MSE }}$ as it is underlined by circles in Fig.11a (i.e. 0.006 for $\eta_{\mathrm{MKE}}$ again 0.014 for $\eta_{\mathrm{MSE}}$ ). The pressure modeshape is significant at the equivalent fluid location but its spatial variations there are small. Therefore the modal kinetic energy is small, thus $\eta_{\mathrm{MKE}}$ is small. This is consistent with the fact that the acoustic velocity for this mode is low and the viscous drag is low at the location of the absorbing material.

Fig. $11 \mathrm{~b}$ shows the modal damping loss factors that vary significantly from one mode to another. For instance, for the $1 \mathrm{kHz}$ third-octave band, the highest damping value occurred for the mode at $946 \mathrm{~Hz}$ (indicated by a cross in Fig. 11b). It presents a mode shape with strong variations of the pressure amplitudes in the fibrous material domain (inducing a high acoustic velocity tangential to the wall) as seen in Fig. 12b, which explains why it is strongly damped by the viscous effect in the fibrous material. On the contrary, the mode at $1003 \mathrm{~Hz}$ has a relatively uniformly distributed spatial shape inside the cavity, as seen in Fig.12c . The modal (kinetic and strain) energies are spread inside the entire volume, and not in particular inside the absorbing material. Consequently, this mode is relatively weakly influenced by the presence of the dissipative material. It presents the lowest damping values of the third octave as it is underscored by a second cross in Fig. $11 \mathrm{~b}$, 


\subsection{SmEdA results}

The final step of the methodology proposed consists in performing the SmEdA calculations, considering a normal point force exerted at point $M_{\mathrm{e}}$, as shown on Fig. 3. This involves calculating the modal injected powers from Eq. (11) and then solving the SmEdA equations (15) depending on the subsystem modal information estimated previously (i.e. modal frequency, mode shapes, and modal damping loss factors).

In this section the SmEdA results are compared between the different configurations.

\subsubsection{Intermodal coupling factors and subsystem modal energies}

The influence of the dissipative material on the SmEdA intermodal coupling factors and on the modal energy distribution for the cavity are illustrated in Fig. 13 , by comparing these quantities between the Bare and Visc 2 configurations. These results concern the $1 \mathrm{kHz}$ third octave band for which the Bare plate and the Visc2 plate have 19 and 18 modes, respectively, whereas the empty cavity has 21 modes. It can be seen that for the Bare case, only two couples of plate-cavity modes $((4,3)$ and $(10,10))$ have significant intermodal coupling factor values. This shows that the coupling strength between these couples of modes is high compared to the other couples. Although the number of subsystem modes is relatively high in this band, few of them participate significantly in the energy sharing between the two subsystems. This is in particular due to the fact that the frequency coincidences play a significant role when the damping is low. On the contrary, for the Visc2 configuration, a large number of couples have significant values for the intermodal coupling factors and participate in the energy sharing between the plate and the cavity. Al- 
most all the cavity modes are significantly coupled to at least one plate mode. As observed in Fig. 13 the result is that the distribution of the modal energies of the cavity is more uniform than for the Bare configuration: a variation of $35 \mathrm{~dB}$ for the Bare configuration compared to $20 \mathrm{~dB}$ for the Visc2 configuration.

Moreover, a considerable decrease of the highest values of the intermodal coupling factors between the two configurations can be seen: $12.5 \mathrm{~Hz}$ for the Bare configuration versus $1.5 \mathrm{~Hz}$ for the Visc2 configuration. In the latter case, more cavity modes are significantly coupled with the plate modes, but with less intensity. These phenomena have the opposite effect on the energy sharing between the two subsystems. Defining which phenomenon is predominant in energy sharing can only be achieved by analysing the final result.

\subsubsection{Subsystem energy}

Subsystem energies are finally obtained by summing the modal energies of each subsystem. Fig. 14 gives these subsystem energies for each third octave band and for each configuration. Note that the SmEdA analysis for the Poro case is carried out up to the $5 \mathrm{kHz}$ band according to the available frequency range of the fibrous material characterization.

As can be expected, the fibrous material has little influence on the plate energies. Indeed, the latter are directly excited by the mechanical force and since the cavityplate coupling is weak [32], the behaviour of the cavity does not significantly influence the plate behaviour. For the cavity, if we exclude the first band (which is particular as the cavity has only 2 modes), it can be seen that the fibrous material reduces the energy from $1.5 \mathrm{~dB}$ to $8 \mathrm{~dB}$ as a function of the frequency band. These reductions should be compared to the variation of the modal damping loss factors of the cavity between the Bare and Poro configuration (see Fig. 11b). Although the 
damping loss factors of many modes of the Poro configuration are ten times higher than those of the Bare configuration, the energy reduction is less than $10 \mathrm{~dB}$. This is due to the fact that the mostly damped cavity modes correspond to localized modes in the fibrous material (as shown in fig. 12 ) and do not participate in the energy sharing between the plate and the cavity. This is illustrated by the mode shown in Fig. $12 \mathrm{~b}$ which presents a high damping loss factor but low intermodal coupling loss factors with the plate modes (values between $8 \cdot 10^{-6} \mathrm{~Hz}$ and $0.08 \mathrm{~Hz}$, whereas the highest value for the different couples of modes is $3.1 \mathrm{~Hz}$ for the $1 \mathrm{kHz}$ third octave band). Using SmEdA to describe the behaviour of each mode also allows describing these phenomena.

The energy difference between the Bare and the Viscl configuration decreases with frequency (around $12 \mathrm{~dB}$ at $315 \mathrm{~Hz}$ and reaches $6 \mathrm{~dB}$ at $8 \mathrm{kHz}$ ). This decrease as a function of the frequency induced by the damping pad can be directly linked to the decrease of the modal damping loss factors of the Viscl plate observed in Fig. 8 . Adding a supplementary damping pad (Visc2 configuration) permits a supplementary decrease of cavity energy of around $3 \mathrm{~dB}$. This decrease can be linked to the increase of around $50 \%$ of the plate modal damping loss factors between the Viscl and Visc 2 configurations, as observed in Fig. 8. Fig. 15 shows the subsystem energy ratios $\left(E_{2} / E_{1}\right)$ for the different configurations. This allows us to characterize the energy transmission between the plate and the cavity. Few differences can be seen between the Bare, Viscl and Visc2 configurations between $2 \mathrm{kHz}$ and $5 \mathrm{kHz}$. Although the damping pads have considerable opposite effects on the modal coupling strengths (as discussed in section 4.5.1), it can be seen here that these effects have a tendency to cancel each other in these frequency bands. Above $5 \mathrm{kHz}$, the energy ratio increases, in particular for the damped cases. The frequency bands concerned are closer to the critical frequency of the plate (around $11 \mathrm{kHz}$ ). This explains why the plate radiates more significantly into the cavity, and why the energy ratio in- 
creases. Below $2 \mathrm{kHz}$, it is more difficult to establish a tendency between the Bare, Visc1 and Visc2 configurations. As the modal densities are low, the energy ratio is strongly influenced by the frequency coincidences, and then the natural frequency of each mode. For the Poro configuration, the energy ratio is between 1.5 and $8 \mathrm{~dB}$ lower than for the bare configuration (except for the first band). This is mainly due to the fact that the poroelastic material dissipates energy inside the cavity but has little influence on the plate behaviour.

\section{Experimental Validation}

In this section, the SmEdA results are compared to the experimental results for the different configurations. After describing the experimental set-up, two types of comparison are made:

- the first addresses the modal damping loss factors, which constitute intermediary data of the SmEdA methodology proposed. The modal damping loss factors obtained with Eq. (30) and (35) are compared with those obtained experimentally by a recently developed high resolution technique [39];

- the second addresses the noise radiated by the plate into the cavity. The energy ratios calculated by SmEdA are compared with those estimated experimentally from several point measurements.

\subsection{Experimental setup}

As shown in figure 17, the cavity was bounded by $7-\mathrm{cm}$ thick walls made of concrete, and by a steel plate. The dimensions of the plate and the cavity are the same as those used in the simulations (see section 4.1.1). The plate is fixed at its bound- 
aries $(2 \mathrm{~cm}$ wide) with four metal bars screwed to the top edges of the cavity. A stationary harmonic transverse force was exerted on the plate using a shaker at the same point considered in the numerical models. The measurements were performed for two frequency domains as a function of the excitation spectrum. A sweep signal was used for the low domain from $1 \mathrm{~Hz}$ to $3.2 \mathrm{kHz}$ with a resolution $0.5 \mathrm{~Hz}$ and the high domain from $3.2 \mathrm{kHz}$ to $10 \mathrm{kHz}$ with a resolution $1.5625 \mathrm{~Hz}$. The force was measured with an impedance head at the point of excitation.

A Polytec laser scanning vibrometer (OFV-3001 controller, OFV-056 scanning head and PSV-Z-040-H junction box) was used to measure the plate velocity at 120 points. The B\&K electrodynamic mini-shaker Type 4810 and the scanning device were mounted above the plate-cavity system, as seen in Fig. 17a The force was measured using an impedance head PCB288D01.

The cavity pressure was measured with four 1/4 inch PCB 130D21 condenser microphones, mounted on a beam that could be rotated inside the closed cavity by turning the connecting shaft, as shown in Fig. 17b. With this device, 64 microphone positions were considered inside the cavity. The responses measured with the vibrometer and the microphones were normalized with the input force.

The plate acceleration and cavity pressure measurements were treated with a multichannel FFT analyser OROS OR35. All the quantities measured were post-processed with MATLAB.

For each discrete frequency $f_{i}$, the space-averaged quadratic velocity of the plate $\bar{v}_{i}^{2}$ and the space-averaged quadratic pressure inside the cavity $\bar{p}_{i}^{2}$ were estimated 
from the mean of the measured point quadratic velocities and from the measured point quadratic pressures, respectively.

Finally, the subsystem energies for each third octave band $f_{1 / 3}$ were estimated by using the relations [10]:

$$
\begin{aligned}
& E_{1}=\rho h L_{x} L_{y} \sum_{i / f_{i} \in f_{1 / 3}} \bar{v}_{i}^{2} \delta f \\
& E_{2}=\frac{L_{x} L_{y} L_{z}}{\rho_{\text {air }} c_{\text {air }}^{2}} \sum_{i / f_{i} \in f_{1 / 3}} \bar{p}_{i}^{2} \delta f
\end{aligned}
$$

where $\delta f$ is the frequency resolution.

\subsection{Comparison of the modal damping loss factors with the experiment}

The modal damping loss factors of the plate and the cavity were estimated experimentally for the different configurations. They were obtained from the time responses measured on each separated subsystem and using the high-resolution modal analysis method [39] based on the ESPRIT algorithm. The details of this method are given in Appendix A. For this experiment, the plate corresponding to each configuration (i.e. Bare, Visc1, Visc2) was excited by an impact hammer PCB 086E80 and the time responses were measured at several locations with a piezoelectric $B \& K$ accelerometer type 4508 . Furthermore, the top opening of the cavity was closed with a $5 \mathrm{~cm}$ concrete slab (to replace the steel plate and avoid flanking energy transmission) and excited by a loudspeaker with a white noise spectrum. The pressure decays were measured as the sound source was turned off. Three positions of the loudspeaker and two microphone locations per source position were considered for the Bare and Poro configurations.

Fig. 18 presents the experimental modal damping loss factors in comparison with the numerical ones obtained with Eq. (30) for the plate and Eq. (35) for the cavity 
(see sections 3.3. A zoomed view of the high frequency domain of Fig. 18c is proposed in Fig. 19 in order to facilitate the comparison for this region presenting a high modal density for the cavity. Good agreements can be observed in general when taking into account the fact that the ESPRIT method permits estimating only the damping loss factors of the lightly damped modes. Indeed, the noise to signal ratio is not high enough to extract the modal information in the time signal if the mode is strongly damped or if it does not participate significantly in the response at the points measured. This is highlighted in particular for the cavity modes with the poroelastic material for which numerous modes localized in the material are strongly damped and do not participate in the response at the points measured (outside the material). This explains why only a few values of the modal damping loss factors were estimated experimentally in comparison to the numerical values (see Fig. 18c. In conclusion, for these cases the good agreement between the calculations and the measures allowed us to validate the numerical process used to estimate the modal damping loss factors of the subsystems and the effect of dissipative materials. Moreover, we underline that regarding these comparisons, the assumption of neglecting the cross-modal terms appears acceptable in order to approximate the modal damping loss factors.

\subsection{Comparison of the subsystem energy ratios with the experiment}

Fig. 20 compares the numerical and experimental energy ratios for the different configurations. Globally good agreement can be observed above $1 \mathrm{kHz}$ whereas some discrepancies can be noticed below $1 \mathrm{kHz}$ whatever the configuration. SmEdA is based on the coupling of resonant modes excited by broadband forces. For low frequency predictions, two difficulties appear because of reduced modal density 
but also small frequency band excitation. At least one mode has to be resonant in the band and the coupling coefficient can be badly estimated due to calculations assuming a large excitation band. The method appears applicable down to the third octave $315 \mathrm{~Hz}$ (see table (2)). The discrepancies observed are likely due to the fact that the experimental plate boundary conditions do not strictly correspond to the clamped boundary conditions considered in the calculations. The result is that the spatial and spectral couplings between the subsystem modes are significantly different. This effect is important only in the low frequency bands because of small number of resonant modes. Because of averaging, the higher the frequency, the lower the effect of the boundary conditions, as observed in the earliest developments of the SEA method [10]. To improve prediction with the present approach, larger frequency bands can be considered instead of the third octave. It will be also possible to couple SmEdA with an uncertainty quantification technique in order to quantify the variance of the energy response, in particular to boundary conditions. This work is however outside the scope of the present paper and will require further investigations for which the papers of [11,56] will serve as starting points.

At $8 \mathrm{kHz}$, a $5 \mathrm{~dB}$ discrepancy can be observed for the Visc configurations. We associate this discrepancy with a non-modal behaviour of the panel due to considerable damping (i.e. high modal overlap). The direct field may be significant compared to the reverberant field and it cannot be correctly predicted by SEA type methods [14,57].

The SmEdA methodology gives a good estimation of the energy transmission between the panel and the cavity between these two frequency domains for which the subsystem behaviour is weakly sensitive to the boundary conditions and dominated by the reverberant field. It is noteworthy that for the validation, the test case was designed to stay as close as possible to the assumptions of the methodology pro- 
posed, in particular the assumptions relating to the use of the equivalent models. The steel panel is flat with no increase in thickness and the viscoelastic layer is thin. On the other hand, the porous material was placed on a rigid surface and presented a sufficiently high porosity to neglect the effect of the skeleton. In addition the use of the equivalent fluid model was well adapted. Apart from these considerations, the present comparisons clearly validate the application of the methodology proposed on the laboratory test case considered. In the conclusions of the present paper, and the applicability and limitations of the approach proposed will be discussed for predicting the viboacoustic behaviour of industrial structures including viscoelastic layers and porous materials.

\section{Conclusions}

A methodology was proposed in the framework of SmEdA for predicting the effect of dissipative materials (i.e. viscoelastic layer patches, poroelastic materials) on the vibro-acoustic behaviour of complex mechanical structures. The SmEdA model was based on Finite Element models of the uncoupled-subsystems, taking the dissipative treatments into account through homogenized material models that facilitate their introduction in FE models. This also reduced computing times in comparison to a full model, a feature that is important for re-analysis in view to optimization. A key point of this approach relied on the use of the Galerkin procedure with the normal modes for estimating the modal damping loss factors of the subsystems treated. These factors expressed the energy dissipation in the SmEdA model and depended on the characteristics and the position of the dissipative material in the subsystems treated. It should be recalled that the resulting cross-modal coupling terms associated with subsystem heterogeneities were neglected. Hence, the modal damping loss factors used were the results of an approximation. 
The present methodology was applied to a plate-cavity test case with different configurations of dissipative treatments. Two types of comparisons with experiments were carried out:

- the first concerned the modal damping loss factors which were compared with experimental values measured by a high-resolution modal technique. Although some off-diagonal terms of the modal damping matrix may appear significant compared to the diagonal terms (especially for the cavity with the absorbing material), good agreements between the calculations and measurements were observed, thereby validating the equivalent models for these cases and the use of the Galerkin procedure with normal modes for estimating the modal damping loss factors;

- The second consisted in comparing the energy ratios predicted and those estimated from point measurements. Discrepancies appeared below $1 \mathrm{kHz}$, which can be linked to the plate boundary conditions. Good agreement above $1 \mathrm{kHz}$ was observed for energy transmissions predicted by SmEdA in different configurations of added damping patches and absorbing materials.

For the purposes of validation, the methodology proposed was applied to a test case conforming to the assumptions of the equivalent homogenized models. We expect to widen its applicability to more complex systems, which is necessary in an industrial environment, for instance, to model the vibroacoustic behaviour of a passenger car or a truck cabin, including the effect of trims and damped treatments. The use of subsystem modal information estimated by the finite element method as input data of our numerical process allows us to deal with systems with complex geometries and mechanical properties with spatial variations. However, some issues were identified and have to be addressed attentively:

- As underlined in this paper, an important assumption of the present approach 
consists in neglecting the cross-modal terms in the results of the Galerkin method. This assumption was correctly complied with in the cavity-panel case and it can be expected to be valid when the dissipative treatment is lightly damped and covers a large part of the system considered, even if the system has a complex geometry. However, investigations should be carried out in the future to define practical criteria to ensure the validity of this assumption;

- The reliability of the finite element modelling of subsystems including the dissipative treatments has to be considered. Attention should be given to the use of the equivalent models for the dissipative treatments. The equivalent multi-layer model considered in the present paper can be expected to be a good approximation for modelling slightly curved panels with added thin viscoelastic layers. For instance, for truck applications, when the viscoelastic patches are fixed on a relatively flat part of the cabin structure, the model may be assumed to be adapted. On the contrary, if the pads are fixed on ribs or very curved parts, the equivalent model considered will be not adapted. For these cases, the use of 3-dimensional solid elements to represent the viscoelastic materials in the finite element model may be more appropriate. Likewise, the equivalent fluid model of the porous material is relevant when the solid phase deformation of the material response can be neglected. This is the case, for instance, when the bulk modulus of the porous skeleton is close to zero. This situation is often encountered in car and truck applications since light weight fibrous materials are commonly used. On the contrary, modelling a soundproofing material including a heavy mass and an acoustic spring layer can be problematic. The use of a Biot model for modelling the stiffness of the acoustic layer may be required. However, in this case, further developments would be necessary to adapt the process for estimating the modal damping loss factors with the Galerkin method.

Works are currently in progress to apply the approach proposed to predict sound 
radiated into a truck cabin, including dissipative treatments when the floor is excited by normal point forces. Comparisons with experimental measurements are being carried out and several intermediate results can be found in [31].

\section{Acknowledgments}

This work was funded jointly by the French Government (FUI 12 - Fonds Unique Interministériel) and the European Union (FEDER - Fonds européen de développement régional). It was carried out in the framework of the LabEx CeLyA ("Centre Lyonnais d'Acoustique", ANR-10-LABX-60) and the research project CLIC ("City Lightweight Innovative Cab") bearing the label of the LUTB cluster ("Lyon Urban Truck \& Bus"). We are very grateful to Pascal Vouagner and Cécile Deslot of ACOEM for having characterized the viscoelastic layers with the dynamic mechanical analyser and to Fabien Acher of Volvo Trucks for his valuable comments on the vibro-acoustic modelling aspects of truck applications.

\section{Appendix A Modal damping loss factor estimations - ESPRIT algorithm}

In order to precisely identify the experimental damping loss factors of the damped plates and cavity up to the high frequency domain with high modal overlap, we used a high-resolution modal analysis technique [39] based on the ESPRIT algorithm [38]. This high-resolution method assumes that the signal $s(t)$ is a sum of complex exponentials $x(t)$ (the modal signal to be determined) and white noise $\beta(t)$ :

$$
s(t)=x(t)+\beta(t)=\sum_{k=1}^{K} a_{k} e^{-\alpha_{k} t} e^{j\left(2 \pi f_{k} t+\phi_{k}\right)}+\beta(t)=\sum_{k=1}^{K} b_{k} z_{k}^{t}+\beta(t)
$$


where $K$ is the number of complex exponentials, $b_{k}=a_{k} e^{j \phi_{k}}$ are the complex amplitudes (with $a_{k}$ and $\phi_{k}$ the modal amplitudes and phases at the point of interest), and $z_{k}=e^{-\alpha_{k} t} e^{j 2 \pi f_{k}}$ the so-called poles (with $f_{k}$ the modal frequencies in $\mathrm{Hz}$ and $\alpha_{k}$ the modal damping factors in $\mathrm{s}^{-1}$ ). The modal damping factor $\alpha_{k}$ (also called modal decay constant in $\mathrm{s}^{-1}$ ), the modal decay time $\tau_{k}$ (in s) and the modal damping loss factor $\eta_{k}$ (dimensionless) are linked to each other as follows:

$$
\alpha_{k}=\frac{1}{\tau_{k}}=\frac{\eta_{k} \omega_{k}}{2} \quad, \quad \eta_{k}=\frac{\Delta f_{k,-3 \mathrm{~dB}}}{f_{k}}=\frac{\alpha_{k}}{\pi f_{k}}
$$

where $\omega_{k}$ is the modal angular frequency (in rad.s ${ }^{-1}$ ) and $\Delta f_{k,-3 \mathrm{~dB}}$ the half-power modal bandwidth. The rotational invariance property of the signal subspace (see Roy et al. [38] for mathematical developments) is used to estimate the modal parameters: frequencies, damping factors and complex amplitudes. The dimensions of both subspaces - the signal subspace (spanned by the sinusoids) and the noise subspace (its supplementary) - must be chosen beforehand and the quality of the estimation greatly depends on the correct choice of these parameters. The best choice for the dimension of the signal subspace is the number of complex exponentials actually present in the signal. This number $(K)$ is twice the number of real decaying sinusoids (modes). Prior to the modal analysis itself, an estimate of this number is obtained using the ESTER (ESTimation ERror) technique [58] which consists in minimizing the error on the rotational invariance property of the signal subspace spanned by the sinusoids. The three main steps of the high-resolution method are: (a) reconstruction of the acceleration impulse response; (b) signal conditioning (band-pass filtering, downsampling, etc.) as proposed by Laroche [59]; (c) order detection and determination of modal parameters. More details on the different steps of the method are given in Ege et al. [39], where the method is validated on measured and synthesized signals for the frequency domain where the Fourier 
transform meets its limits (due to high modal overlap or a poor signal-to-noise ratio). Recently, the method has also been successfully applied to orthotropic ribbed plates [60] and multilayer sandwich composites [61].

\section{References}

[1] C. T. Musser, J. E. Manning, G. C. Peng, Predicting vehicle interior sound with statistical energy analysis, in: Proceedings of SAE 2011 Noise and Vibration Conference and Exhibition, Grand Rapids, MI, USA, 2012.

[2] A. Duval, J. Baratier, C. Morgenstern, Trim fem simulation of a dash and floor insulator cut out modules with structureborne and airborne excitations, in: Proceedings of Acoustics 08, Paris, France, 2008.

[3] A. Charpentier, S. Prasanth, F. Kazuki, Efficient model of structure-borne noise in a fully trimmed vechicle from $200 \mathrm{hz}$ to $1 \mathrm{khz}$, in: Proceeding of InterNoise, Shanghai, China, 2008.

[4] C. Felippa, Introduction to finite element methods, University of Colorado, Boulder, USA, 2001.

[5] N. Alam, N. Asnani, Vibration and damping analysis of multilayered rectangular plates with constrained viscoelastic layers, Journal of Sound and Vibration 97 (1984) $597-614$.

[6] M. A. Biot, Generalized theory of acoustic propagation in porous dissipative media, Journal of the Acoustical Society of America 34 (1962) 1254-1264.

[7] C. J. Cameron, P. Wennhage, P. Goransson, Prediction of nvh behavior of trimmed body components in the frequency range 100-500 hz, Applied Acoustics 71 (2010) $708-721$. 
[8] A. Craggs, Coupling of finite element acoustic absorption models, Journal of Sound and Vibration 66 (4) (1979) 605-613.

[9] A. Le Bot, Foundation of statistical energy analysis in vibroacoustics, Oxford university press, 2015.

[10] R. H. Lyon, R. G. Dejong, Theory and Application of Statistical Energy Analysis, Butterworth-Heineman, 1995.

[11] R. S. Langley, V. Cotoni, Response variance prediction in the statistical energy analysis on built-up systems, Journal of the Acoustical Society of America 115 (2) (2005) 706718.

[12] D. Rhazi, N. Atalla, Transfer matrix modeling of the vibroacoustic response of multimaterials structures under mechanical excitation, Journal of the Acoustical Society of America 329 (13) (2010) 2532-2546.

[13] J. Allard, N. Atalla, Propagation of Sound in Porous Media: Modelling Sound Absorbing Materials, Wiley, Chichester, UK, 2009.

[14] A. Le Bot, V. Cotoni, Validity diagrams of statistical energy analysis, Journal of sound and vibration 329 (2010) 221-235.

[15] A. Culla, A. Sestieri, Is it possible to treat confidentially sea the wolf in sheep's clothing?, Mechanical systems and signal processing 20 (2006) 1372-1399.

[16] E. Deckers, O. Atak, L. Coox, R. D’Amico, H. Devriendt, S. Jonckheere, K. Ku, B. Pluymers, D. Vandepitte, W. Desmet, The wave based method: an overview of 15 years of research, Wave Motion 51 (2014) 550-565.

[17] E. Deckers, A wave based approach for steady-state biot models of poroelastic materials, Ph.D. thesis, KU Leuven, Leuven, Belgium (2012).

[18] S. Jonckheere, Wave based and hybrid methodologies for vibro-acoustic simulation with complex damping treatments, Ph.D. thesis, KU Leuven, Leuven, Belgium (2014). 
[19] P. Shorter, R. Langley, Vibro-acoustic analysis of complex systems, Journal of Sound and Vibration 288 (3) (2005) 669-699.

[20] P. J. Shorter, R. S. Langley, On the reciprocity relationship between direct field radiation and diffuse reverberant loading, Journal of the Acoustical Society of America 117 (2005) 85-95.

[21] S. M. Chen, D. F. Wang, J. M. Zan, Interior noise prediction of the automobile based on hybrid fe-sea method, Mathematical Problems in Engineering 2011 (2011) 327170.

[22] A. Charpentier, P. Streedhar, K. Fukui, Using the hybrid fe-sea method to predict structure-borne noise transmission in a trimmed automotive vehicle, SAE paper 200701-2181.

[23] L. Maxit, Reformulation and extension of sea model by relaxing the modal energy equipartition (in french, Ph.D. thesis, Institut National des Sciences Appliquees de Lyon, Lyon, France (2000).

[24] L. Maxit, J.-L. Guyader, Estimation of sea coupling loss factors using a dual modal formulation and fem modal infromation, part i: Theory, Journal of Sound and Vibration 239 (5) (2001) 907-930.

[25] L. Maxit, J.-L. Guyader, Extension of sea model to subsystems with non-uniform modal energy distribution, Journal of Sound and Vibration 265 (2) (2003) 337-358.

[26] F. Cura, A. Mura, F. Scarpa, Modal strain energy based methods for the analysis of complex patterned free layer damped plates, Journal of Vibration and Control 18 (9) (2011) 1291-1302.

[27] T. Yamaguchi, Y. Kurosawa, H. Enomoto, Damped vibration analysis using finite element method with approximated modal damping for automotive double walls with a porous material, Journal of Sound and Vibration 325 (2009) 436-450.

[28] H. Hwang, Extension of the SmEdA method by taking into account dissipative materials in mid-frequency, Ph.D. thesis, INSA Lyon, Lyon, France (2015). 
[29] H. Hwang, K. Ege, L. Maxit, N. Totaro, J.-L. Guyader, A methodology for including the effect of a damping treatment in the mid-frequency domain using SmEdA method, in: Proceedings of 20th International Congress on Sound and Vibration, ICSV20, Bangkok, Thailand, 2013.

[30] H. Hwang, K. Ege, L. Maxit, N. Totaro, J.-L. Guyader, Equivalent damping modeling in the framework of SmEdA, in: Proceedings of XIX-th symposium VIbrations, SHocks \& NOise (VISHNO), Aix-en-Provence, France, 2014.

[31] Y. Gerges, H. Hwang, K. Ege, L. Maxit, C. Sandier, Vibroacoustic modeling of a trimmed truck cab in the mid frequency range, in: Proceedings of Inter-Noise, San Francisco, USA, 2015.

[32] F. Fahy, Vibration of containing structures by sound in the contained fluid, Journal of Manufacturing Science and Engineering 91 (1969) 939-950.

[33] F. Fahy, P. Gardonio, Sound and Structural Vibration, Radiation, Transmission and Response (Second Editon), Academic Press, London, 2006.

[34] J. Sigrist, Fluid-structure interaction, an introduction to finite element coupling, Wiley, 2015.

[35] N. Totaro, C. Dodard, J. L. Guyader, SEA Coupling Loss Factors of Complex VibroAcoustic Systems, Journal of Vibration and Acoustics - Transactions of the ASME 131 (4) (2009) 0410091-0410098.

[36] T. D. Scharton, R. H. Lyon, Power flow and energy sharing in random vibration, Journal of the Acoustical Society of America 43 (1968) 1332-1343.

[37] D. A. Bies, S. Hamid, In situ determination of loss and coupling loss factors by the power injection method, Journal of Sound and Vibration 70 (1980) 187-204.

[38] R. Roy, T. Kailath, Esprit - estimation of signal parameters via rotational invariance techniques, IEEE Transactions on Acoustics Speech and Signal Processing 37 (7) (1989) 984-995. 
[39] K. Ege, X. Boutillon, B. David, High-resolution modal analysis, Journal of Sound and Vibration 325 (4-5) (2009) 852-869.

[40] M. Danti, D. Vigè, G. Nierop, Modal methodology for the simulation and optimization of the free-layer damping treatment of a car body, Journal of Vibration and Acoustics - Transactions of the ASME 132 (2010) 0210011-0210018.

[41] C. D. Johnson, D. A. Kienholz, Prediction of damping in structures with viscoelastic materials, in: MSC/NASTRAN User's Conference Proceedings, 1983.

[42] J.-L. Guyader, Transparence acoustique de plaques multicouches orthotropes, viscoélastiques, finies, Ph.D. thesis, INSA Lyon, France (1977).

[43] J.-L. Guyader, C. Lesueur, Acoustic transmission through orthotropic multilayered plates, part i: plate vibration modes, Journal of Sound and Vibration 58 (1) (1978) $51-58$.

[44] J.-L. Guyader, C. Cacciolati, Viscoelastic properties of single layer plate material equivalent to multi-layer composite plate, in: Proceedings of Inter-Noise, Istanbul, Turkey, 2007.

[45] M. A. Biot, Theory of propagation of elastic waves in a fluid-saturated porous solid. i. low-frequency range, The Journal of the Acoustical Society of America 28 (2) (1956) $168-178$.

[46] A. Craggs, A finite element model for rigid porous absorbing materials, Journal of Sound and Vibration 61 (1978) 101-111.

[47] B. Bécot, F. Sgard, On the use of poroelastic materials for the control of the sound radiated by a cavity backed plate, Journal of the Acoustical Society of America 120 (4) (2006) 2055-2066.

[48] A. Seybert, R. Seman, M. Lattuca, Boundary element prediction of sound propagation in ducts containing bulk absorbing materials, Journal of vibration and acoustics 120 (1998) 976-981. 
[49] J. F. Allard, Propagation of Sound in Porous Media: Modelling Sound Absorbing Materials, Elsevier Applied Science, London, 1993.

[50] O. Doutre, N. Dauchez, J. Génevaux, O. Dazel, Validity of the limp model for porous materials: a criterion based on the biot theory, Journal of the Acoustical Society of America 122 (2007) 2038-2048.

[51] H. Utsuno, T. Tanaka, T. Fujikawa, Transfer function method for measuring characteristic impedance and propagation constant of porous materials, Journal of the Acoustical Society of America 86 (2) (1989) 637-643.

[52] K. Attenborough, Acoustical characteristics of rigid fibrous absorbents and granular materials, Journal of the Acoustical Society of America 73 (3) (1983) 785-799.

[53] J.-P. Mathieu, On damped vibration theory, International Journal of Mechanical Sciences 7 (3) (1965) $173-182$.

[54] M. L. Williams, R. Landel, J. Ferry, The temperature dependence of relaxation mechanisms in amorphous polymers and other glass-forming liquids, Journal of the American Chemical Society 77 (14) (1955) 3701-3707.

[55] M. Delany, E. Bazley, Acoustical properties of fibrous absorbent materials, Applied Acoustics 3 (1970) 105-116.

[56] R. Langley, A. Brown, The ensemble statistics of the energy of a random system subjected to harmonic excitation, Journal of Sound and Vibration 275 (3) (2004) 823846.

[57] T. Lafont, N. Totaro, A. Le Bot, Review of statistical energy analysis hypotheses in vibroacoustics, Proceedings of the Royal Society A 470 (2013) 20130515.

[58] R. Badeau, B. David, R. Richard, A new perturbation analysis for signal enumeration in rotational invariance techniques, IEEE Transactions on Signal Processing 54 (2) (2006) 450-458. 
[59] J. Laroche, The use of the matrix pencil method for the spectrum analysis of musical signals, Journal of the Acoustical Society of America 94 (4) (1993) 1958-1965.

[60] K. Ege, X. Boutillon, M. Rébillat, Vibroacoustics of the piano soundboard: (non)linearity and modal properties in the low- and mid-frequency ranges, Journal of Sound and Vibration 332 (5) (2013) 1288-1305.

[61] K. Ege, V. Henry, Q. Leclère, R. G. Rinaldi, C. Sandier, Vibrational behavior of multilayer plates in broad-band frequency range: comparisons between experimental and theoretical estimations, in: Proceedings of Internoise 2015, San Francisco, United States, 2015. 


\section{List of Figures}

$1 \quad$ Vibrating panel coupled with an acoustic cavity and excited by a $\begin{array}{ll}\text { mechanical point force } & 61\end{array}$

2 Schematic representation of equivalent single layer modelling for a plate treated with viscoelastic materials

3 Schematic representation of the plate-cavity system excited by a point force (Bare configuration).

4 Description of the three configurations of dissipative treatments:

(a) Viscl configuration; (b) Visc2 configuration; (c) Poro configuration.

5 (a) Young's modulus averaged in third octave bands: $\square, E_{\mathrm{ba}}(1 \mathrm{~mm}$ steel plate); $\bigcirc, E_{\text {visc }}(3 \mathrm{~mm}$ viscoelastic layer $)$ and $\diamond, E_{\text {eq }, \mathrm{m}}$ (4 mm equivalent single layer); (b) Component damping loss factor averaged in the third octave band: $\square, \eta_{\mathrm{ba}}(1 \mathrm{~mm}$ steel plate);

$\bigcirc, \eta_{\text {visc }}\left(3 \mathrm{~mm}\right.$ viscoelastic layer) and $\diamond, \eta_{\mathrm{eq}, \mathrm{m}}(4 \mathrm{~mm}$ equivalent single layer)

6 Equivalent fluid parameters of the fibrous material in third octave bands: (a) Density, (b) Compressibility, (c) Damping Loss factors: $\square,-\chi_{\text {eq,f }} ; \bigcirc, \eta_{\text {eq, }}$

7 Absolute values of the modal damping matrix coefficients $\eta_{\mathrm{ba}} \mathbf{W}_{m}^{\mathrm{T}} \overline{\mathbf{K}}_{\mathrm{ba}} \mathbf{W}_{m^{\prime}}+\eta_{\mathrm{eq}, \mathrm{m}} \mathbf{W}_{m}^{\mathrm{T}} \overline{\mathbf{K}}_{\mathrm{eq}, \mathrm{m}} \mathbf{W}_{m^{\prime}}$ (see Eq.(28)): (a), Viscl plate; (b), Visc2 plate. Results for the first ten modes of third octave bands $1600 \mathrm{~Hz}$. 
8 Plate modal damping loss factors as a function of the modal frequency (results of Eq. (30p): $\times$, Visc1 plate; $\bullet$, Visc2 plate. Component damping loss factors by third octave bands: $\square$ : steel plate $; \diamond:$ equivalent viscoelastic multilayer.

9 Example of two modeshapes of plate for the Viscl configuration: (a) At $1584 \mathrm{~Hz}$, (b) At $1828 \mathrm{~Hz}$

10 Absolute values of two modal matrices resulting of the Galerkin method: (a), $\eta_{\text {air }}\left(\mathbf{P}_{n}^{\mathrm{T}} \overline{\mathbf{M}}_{\text {air }} \mathbf{P}_{n^{\prime}}\right)$; (b), $\frac{-\chi_{\text {eq, }}}{1+\chi_{\text {eq, }}^{2}} \frac{\mathbf{P}_{n}^{\mathrm{T}} \overline{\mathbf{K}}_{\text {eq, }} \mathbf{P}_{n^{\prime}}}{\bar{\omega}_{n} \bar{\omega}_{n^{\prime}}}$. Results for the first ten cavity modes of third octave bands $1000 \mathrm{~Hz}$.

11 Cavity modal damping loss factors as a function of the

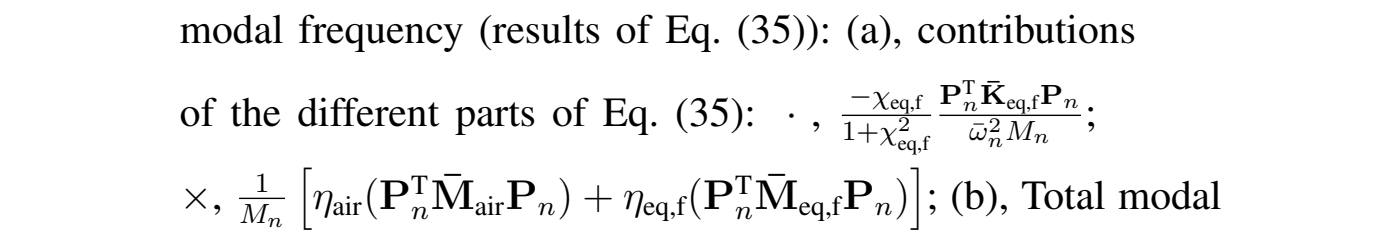
damping loss factors $\left(\bigcirc, \eta_{n}\right)$. ( $\square$, Experimental empty cavity damping averaged in third octave bands)

12 Example of three modeshapes of the cavity for the Poro configuration: (a), $469 \mathrm{~Hz}$;(b), $946 \mathrm{~Hz}$; (c), 1003 Hz. Upside down representation of the cavity for ease visualization.

13 SmEdA results for the third-octave band $1 \mathrm{kHz}$ band (Modes \begin{tabular}{l}
\hline indexed with increasing natural frequencies): (a) and (b), modal \\
\hline coupling loss factors between the cavity modes and the plate \\
\hline modes $\left(\beta_{m n}\right)$; (c) and (d), distribution of modal energy for the \\
\hline cavity (i.e. mode energy in function of the modal order). Two \\
\hline
\end{tabular} configurations: (a) and (c), Bare ; (b) and (d), Visc2. 
14 Subsystem energies in third octave bands for the 4 configurations:

(a) Plate energies, (b) Cavity energies

15 Subsystem energy ratio $E_{2} / E_{1}$ in third octave bands for the 4 configurations: $\star$, Bare configuration; $\square$, Viscl configuration; $\bigcirc$, Visc2 configuration; $\diamond$, Poro configuration

16 Pictures of the different treated subsystems used experimentally:

(a), plate of the Viscl configuration; (b), plate of the Visc2

configuration; (c), cavity with microphones of the Poro

configuration.

17 Experimental setup: (a) Plate-cavity system and the mounted laser vibrometer; (b) Microphones mounted inside the empty cavity; (c) Excitation device.

18 Modal damping loss factors for the treated subsystems: (a), plate of the Viscl configuration; (b), plate of the Visc2 configuration; (c), cavity of the Poro configuration. Comparison of two results: $\bullet$ experimental; $\times$, numerical

20 Subsystem energy ratio in third octave bands for the different configurations: (a), Bare configuration; (b), Viscl configuration; (c), Visc2 configuration; (d), Poro configuration. Comparison of two results: Solid, experimental; dashed, SmEdA prediction 


\section{List of Tables}

1 Mechanical properties of the plate, viscoelastic layer and equivalent plate model. Frequency dependent values given in Fig. 5. (Equivalent parameters computed with MOVISAND software.)

2 Number of subsystem modes in third octave bands for each configuration. 


\section{Figures}

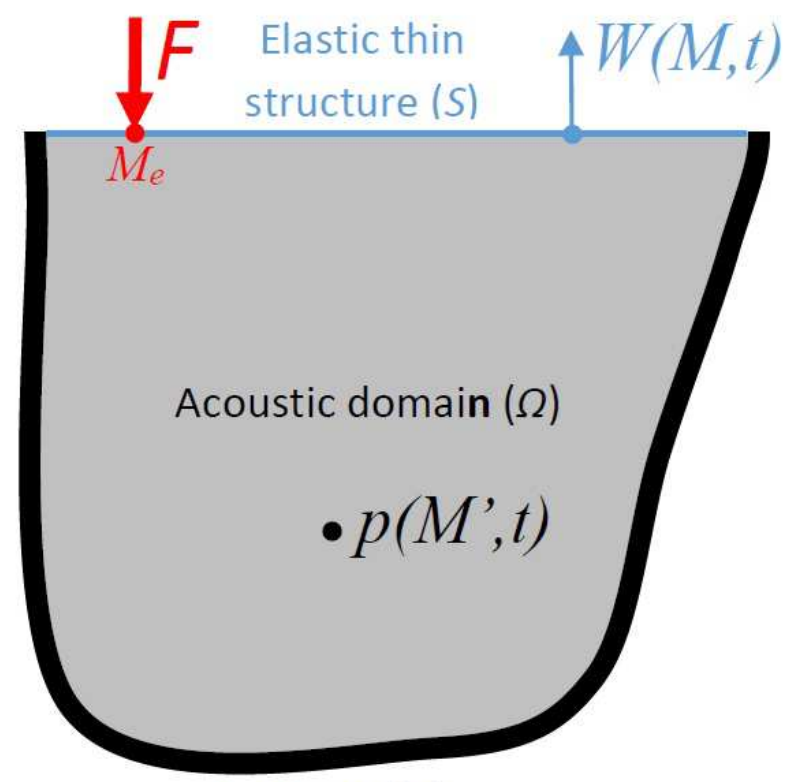

Rigid wall $\left(S_{r}\right)$

Figure 1. Vibrating panel coupled with an acoustic cavity and excited by a mechanical point force

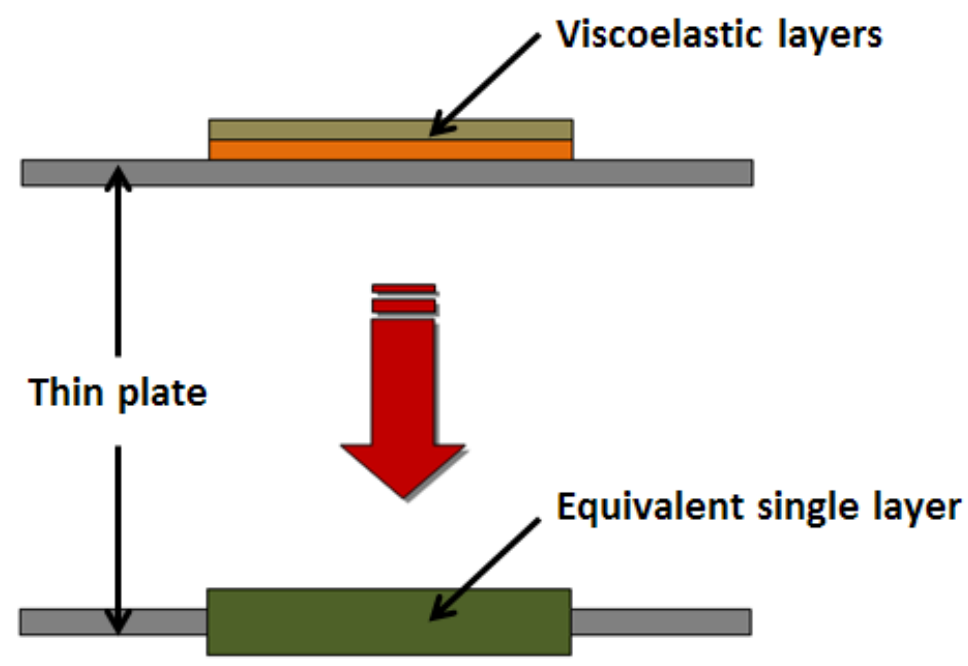

Figure 2. Schematic representation of equivalent single layer modelling for a plate treated with viscoelastic materials 


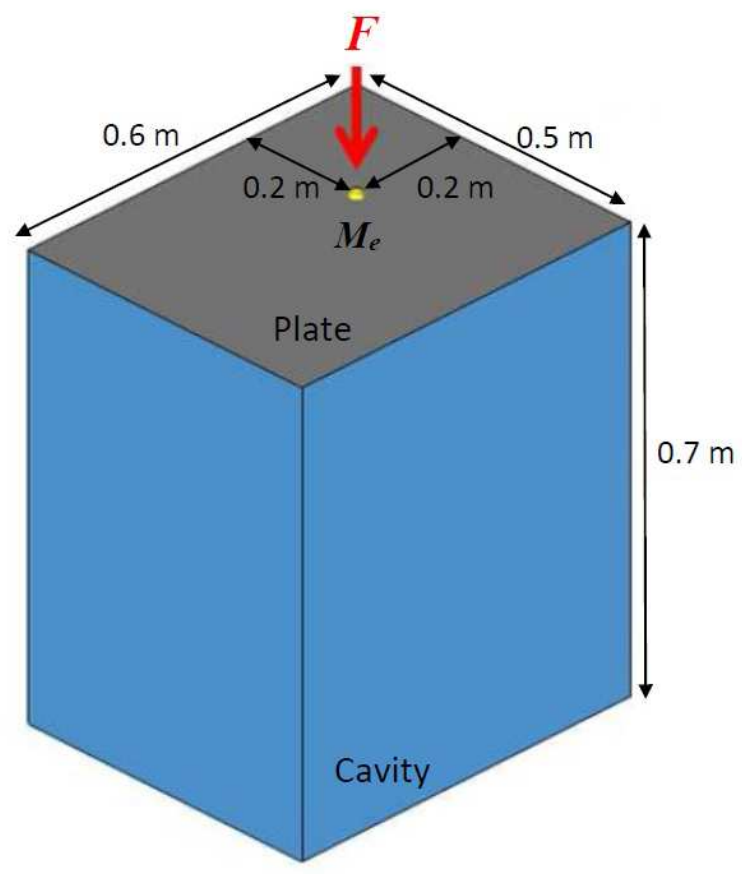

Figure 3. Schematic representation of the plate-cavity system excited by a point force (Bare configuration).

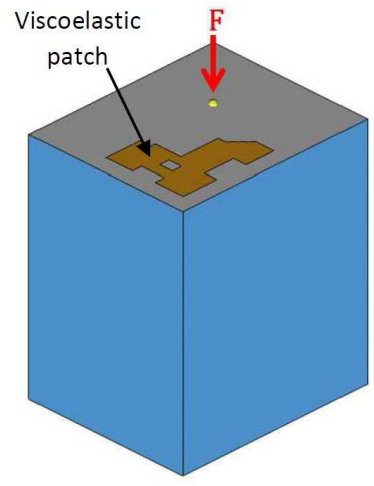

(a)

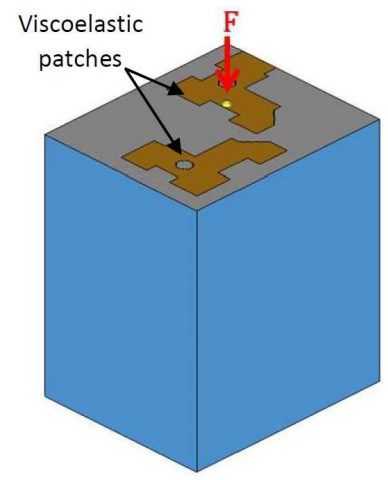

(b)

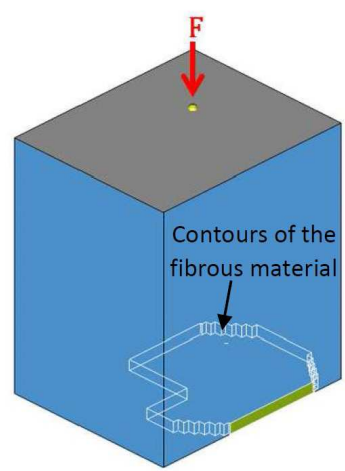

(c)

Figure 4. Description of the three configurations of dissipative treatments: (a) Viscl configuration; (b) Visc2 configuration; (c) Poro configuration. 


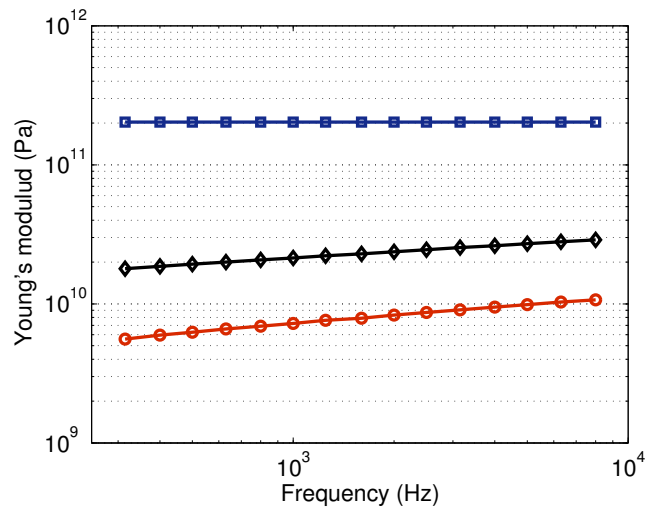

(a)

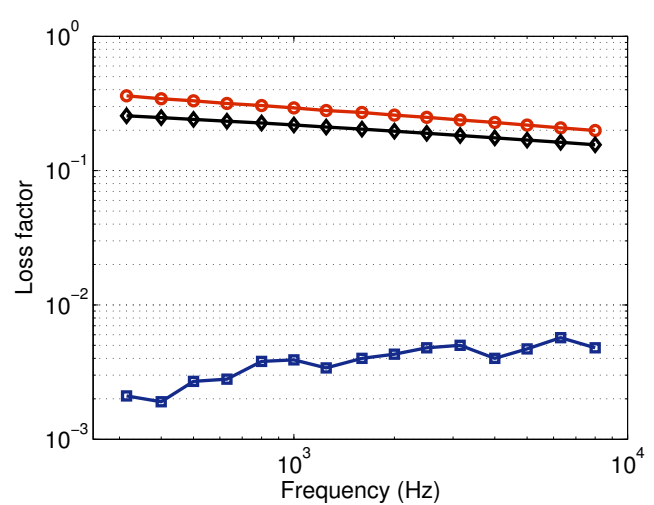

(b)

Figure 5. (a) Young's modulus averaged in third octave bands: $\square, E_{\mathrm{ba}}$ (1 $\mathrm{mm}$ steel plate); $\bigcirc, E_{\text {visc }}$ (3 mm viscoelastic layer) and $\diamond, E_{\text {eq,m }}$ (4 mm equivalent single layer); (b) Component damping loss factor averaged in the third octave band: $\square, \eta_{\mathrm{ba}}(1 \mathrm{~mm}$ steel plate); $\bigcirc, \eta_{\text {visc }}\left(3 \mathrm{~mm}\right.$ viscoelastic layer) and $\diamond, \eta_{\text {eq, } \mathrm{m}}(4 \mathrm{~mm}$ equivalent single layer $)$

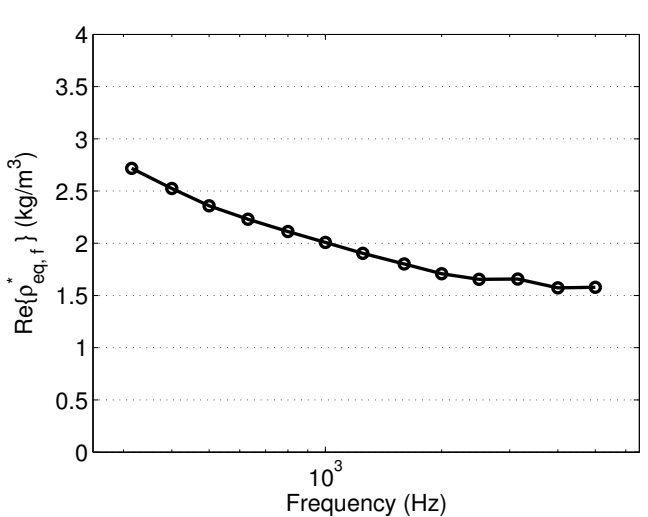

(a)

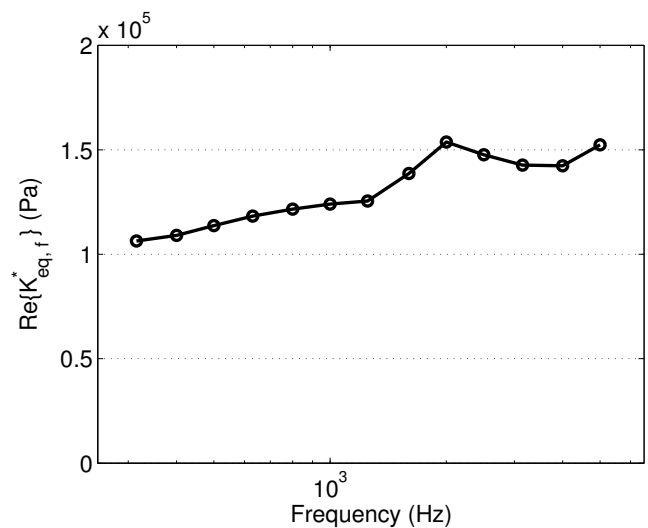

(b)

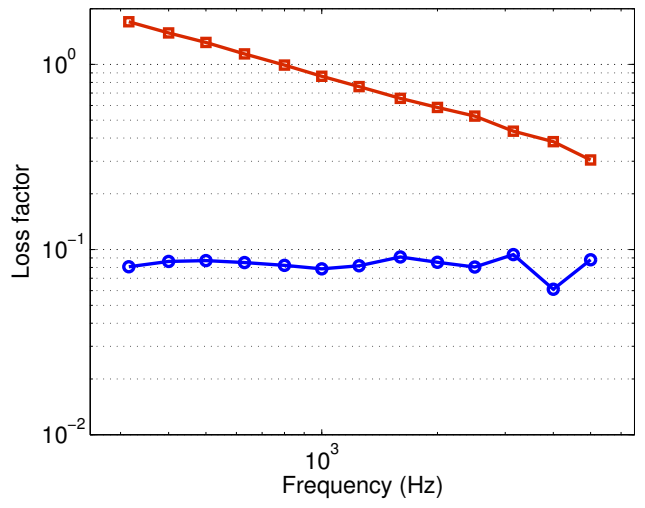

(c)

Figure 6. Equivalent fluid parameters of the fibrous material in third octave bands: (a) Density, (b) Compressibility, (c) Damping Loss factors: $\square,-\chi_{\text {eq, }} ; \bigcirc, \eta_{\text {eq,f }}$ 


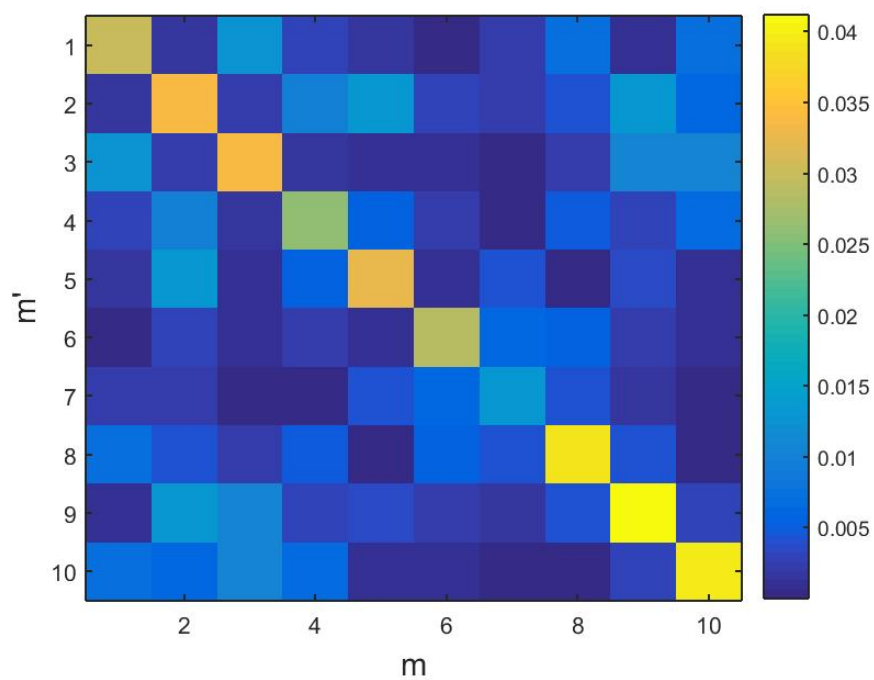

(a)

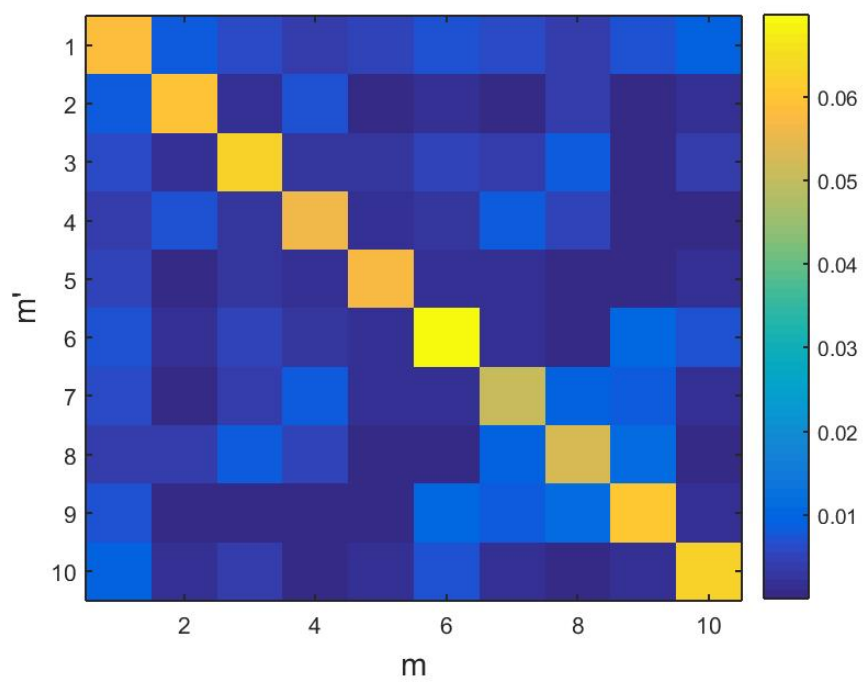

(b)

Figure 7. Absolute values of the modal damping matrix coefficients $\eta_{\mathrm{ba}} \mathbf{W}_{m}^{\mathrm{T}} \overline{\mathbf{K}}_{\mathrm{ba}} \mathbf{W}_{m^{\prime}}+\eta_{\mathrm{eq}, \mathrm{m}} \mathbf{W}_{m}^{\mathrm{T}} \overline{\mathbf{K}}_{\mathrm{eq}, \mathrm{m}} \mathbf{W}_{m^{\prime}}$ (see Eq.28): (a), Visc1 plate; (b), Visc2 plate. Results for the first ten modes of third octave bands $1600 \mathrm{~Hz}$. 


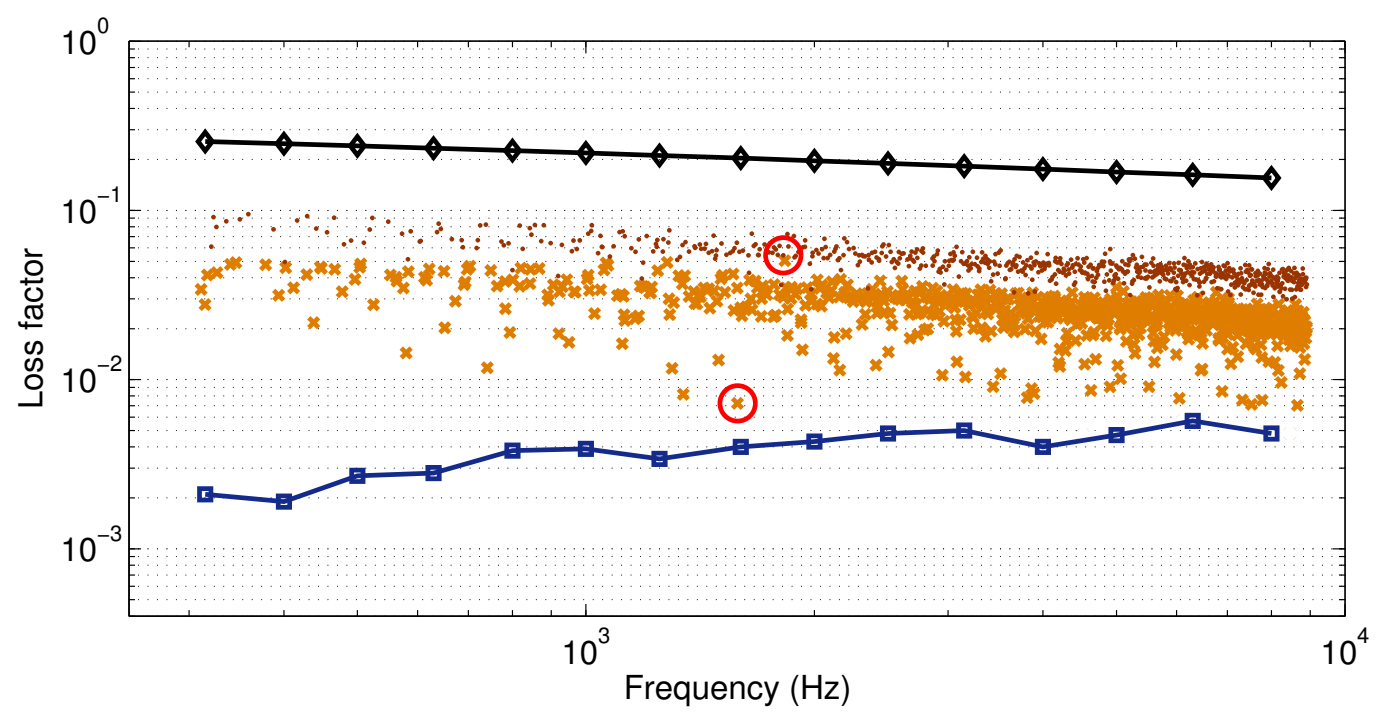

Figure 8. Plate modal damping loss factors as a function of the modal frequency (results of Eq. (30): $\times$, Visc1 plate; $\bullet$ Visc2 plate. Component damping loss factors by third octave bands: $\square$ : steel plate; $\diamond$ : equivalent viscoelastic multilayer.

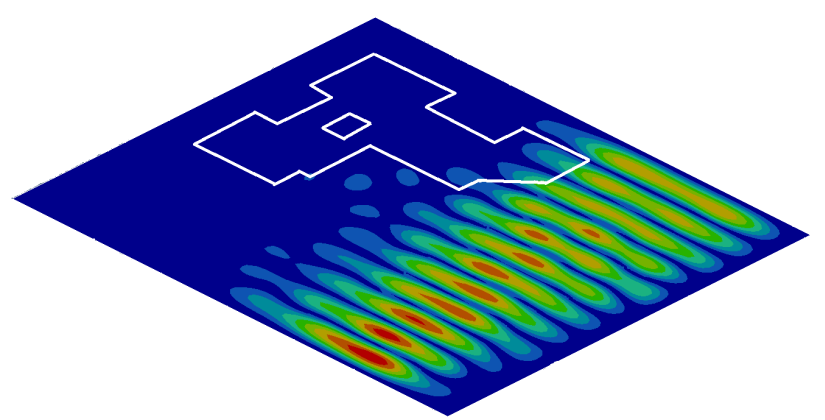

(a)

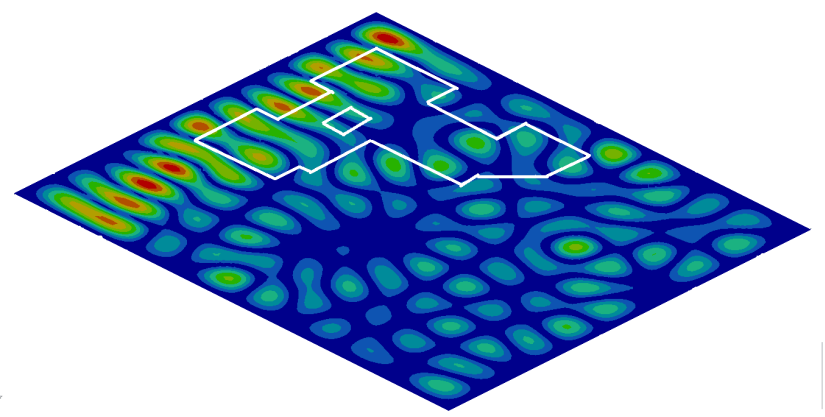

(b)

Figure 9. Example of two modeshapes of plate for the Viscl configuration: (a) At $1584 \mathrm{~Hz}$, (b) At $1828 \mathrm{~Hz}$ 


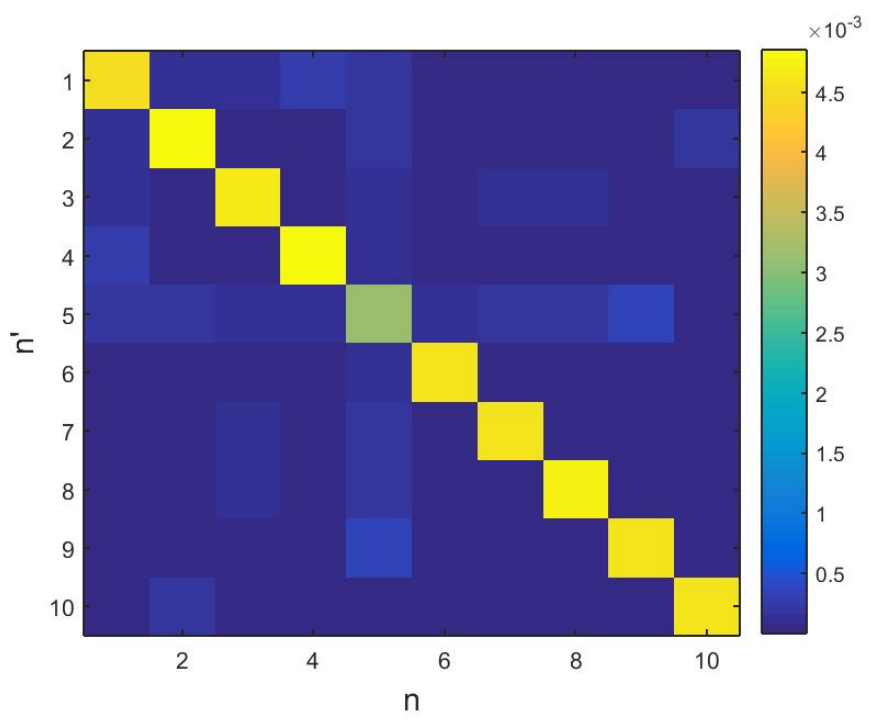

(a)

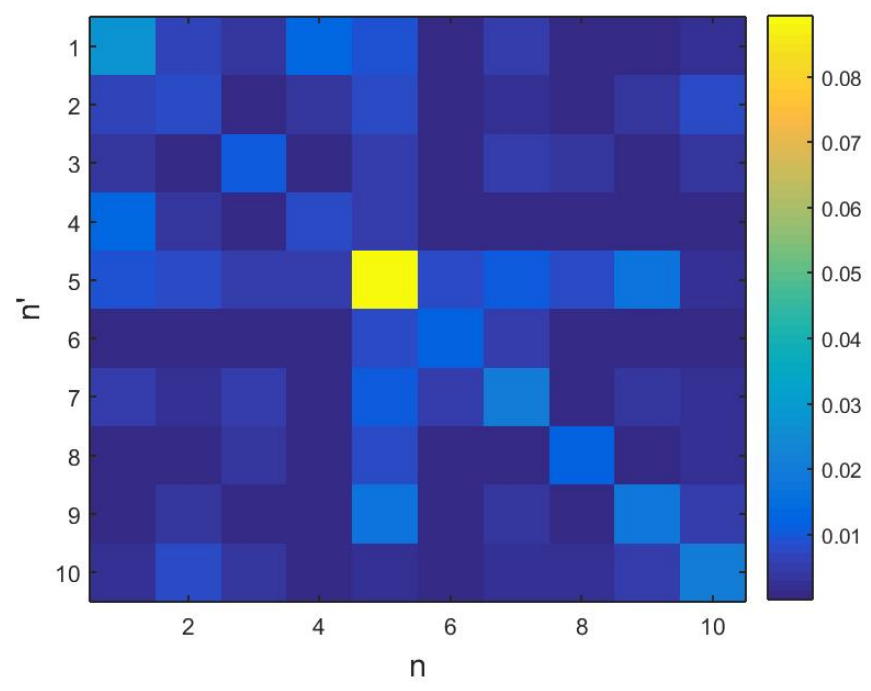

(b)

Figure 10. Absolute values of two modal matrices resulting of the Galerkin method: (a), $\eta_{\text {air }}\left(\mathbf{P}_{n}^{\mathrm{T}} \overline{\mathbf{M}}_{\text {air }} \mathbf{P}_{n^{\prime}}\right)$; (b), $\frac{-\chi_{\text {eq, }}}{1+\chi_{\text {eq, }}^{2}} \frac{\mathbf{P}_{n}^{\mathrm{T}} \overline{\mathbf{K}}_{\text {eq, }} \mathbf{P}_{n^{\prime}}}{\bar{\omega}_{n} \bar{\omega}_{n^{\prime}}}$. Results for the first ten cavity modes of third octave bands $1000 \mathrm{~Hz}$. 


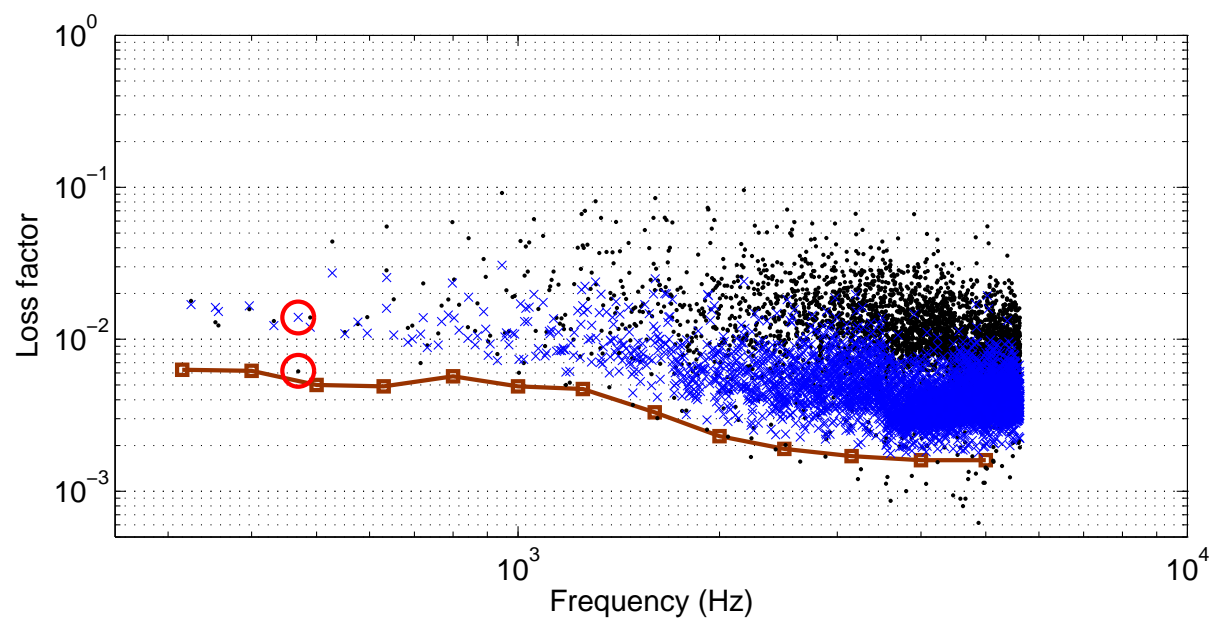

(a)

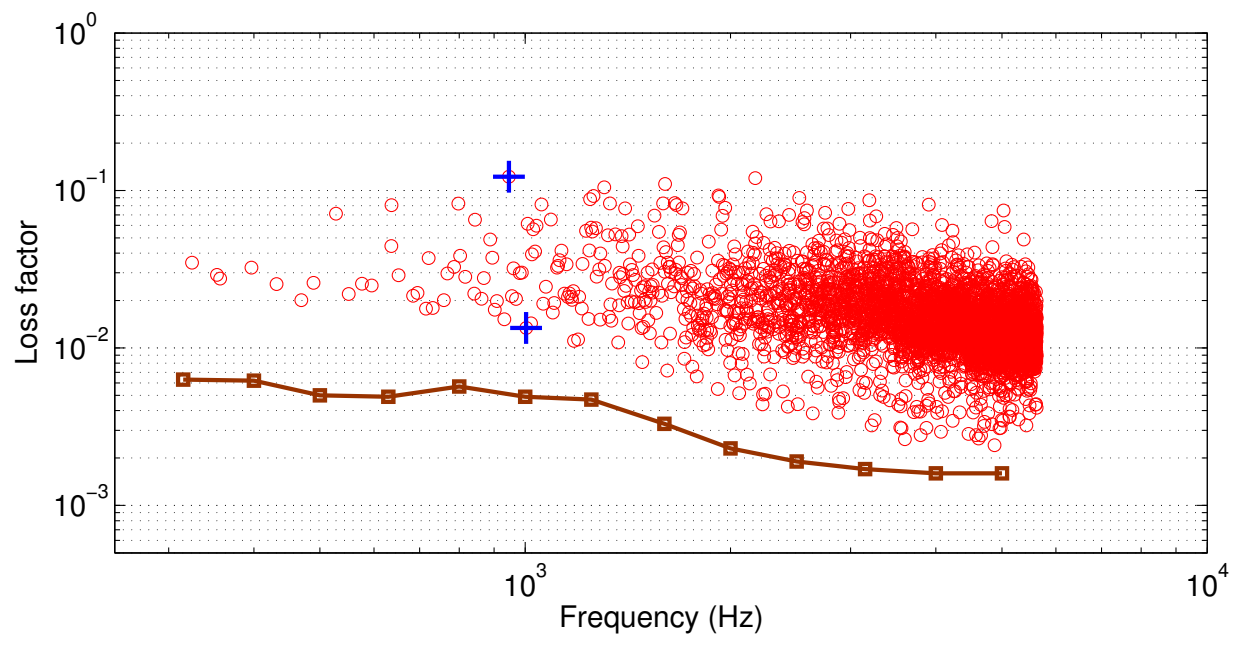

(b)

Figure 11. Cavity modal damping loss factors as a function of the modal frequency (results of Eq. 35 ): (a), contributions of the different parts of Eq. 35 : $\cdot, \frac{-\chi_{\text {eq, }}}{1+\chi_{\text {eq, }}^{2}} \frac{\mathbf{P}_{n}^{\mathrm{T}} \overline{\mathbf{K}}_{\text {eq, }} \mathbf{P}_{n}}{\bar{\omega}_{n}^{2} M_{n}}$; $\times, \frac{1}{M_{n}}\left[\eta_{\text {air }}\left(\breve{\mathbf{P}}_{n}^{\mathrm{T}} \overline{\mathbf{M}}_{\text {air }} \mathbf{P}_{n}\right)+\eta_{\text {eq, }}\left(\mathbf{P}_{n}^{\mathrm{T}} \overline{\mathbf{M}}_{\text {eq, } \mathrm{f}} \mathbf{P}_{n}\right)\right] ;$ (b), Total modal damping loss factors $(\bigcirc$, $\left.\eta_{n}\right)$. ( $\square$, Experimental empty cavity damping averaged in third octave bands) 


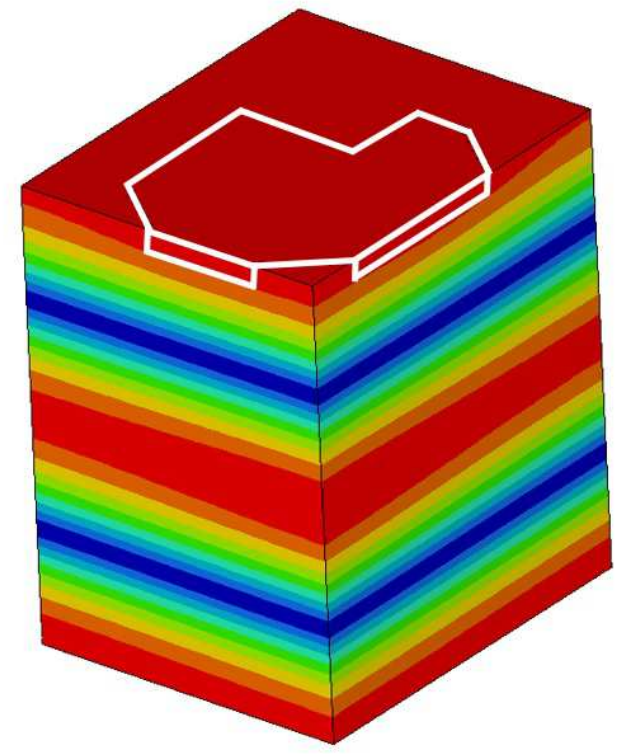

(a)

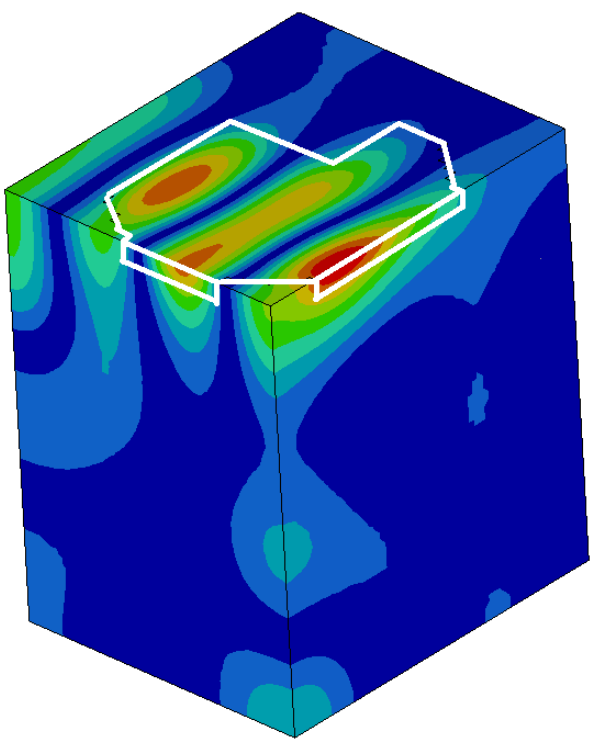

(b)

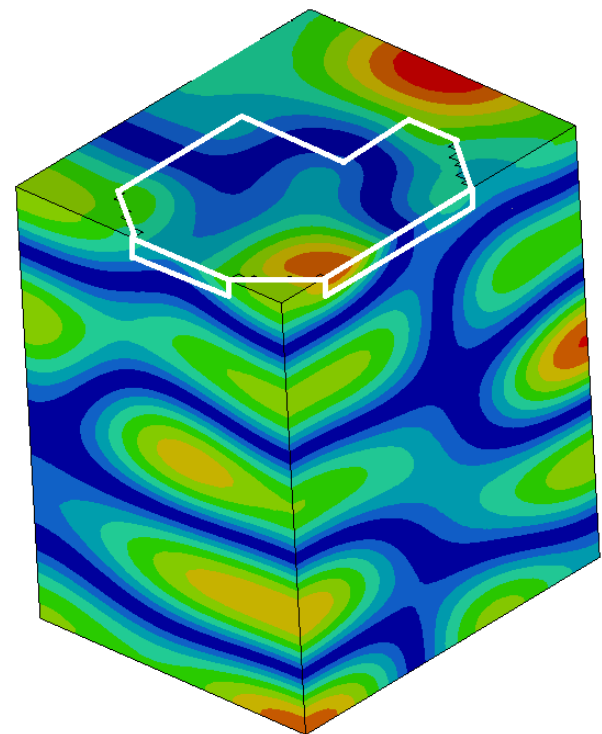

(c)

Figure 12. Example of three modeshapes of the cavity for the Poro configuration: (a), $469 \mathrm{~Hz}$;(b), $946 \mathrm{~Hz}$; (c), $1003 \mathrm{~Hz}$. Upside down representation of the cavity for ease visualization. 


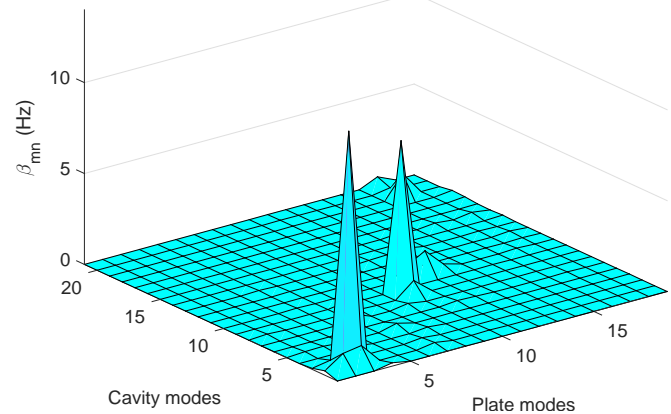

(a)

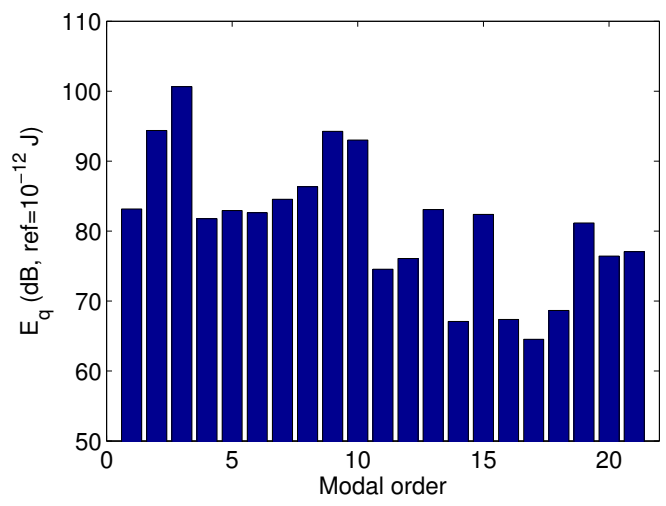

(c)

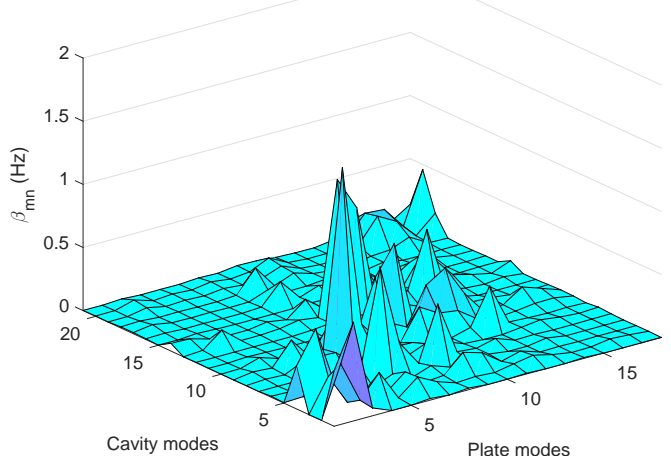

(b)

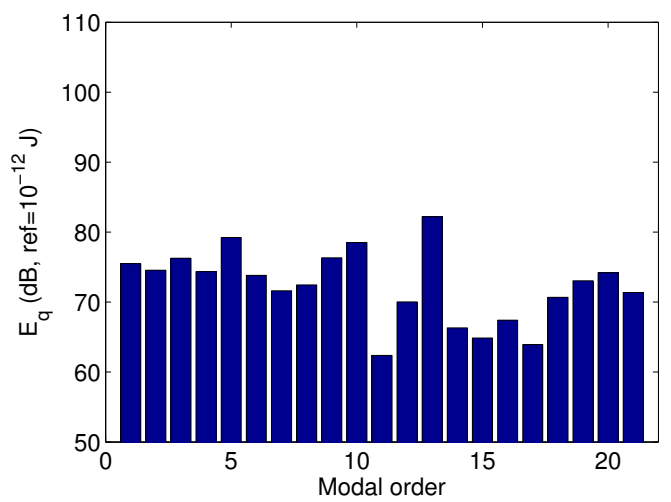

(d)

Figure 13. SmEdA results for the third-octave band $1 \mathrm{kHz}$ band (Modes indexed with increasing natural frequencies): (a) and (b), modal coupling loss factors between the cavity modes and the plate modes $\left(\beta_{m n}\right)$; (c) and (d), distribution of modal energy for the cavity (i.e. mode energy in function of the modal order). Two configurations: (a) and (c), Bare ; (b) and (d), Visc2. 


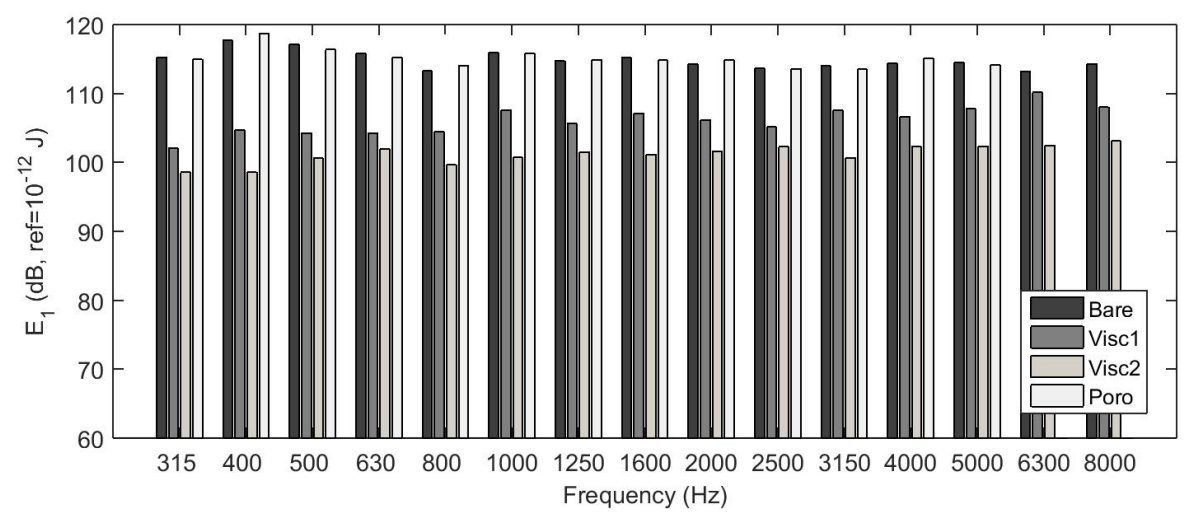

(a)

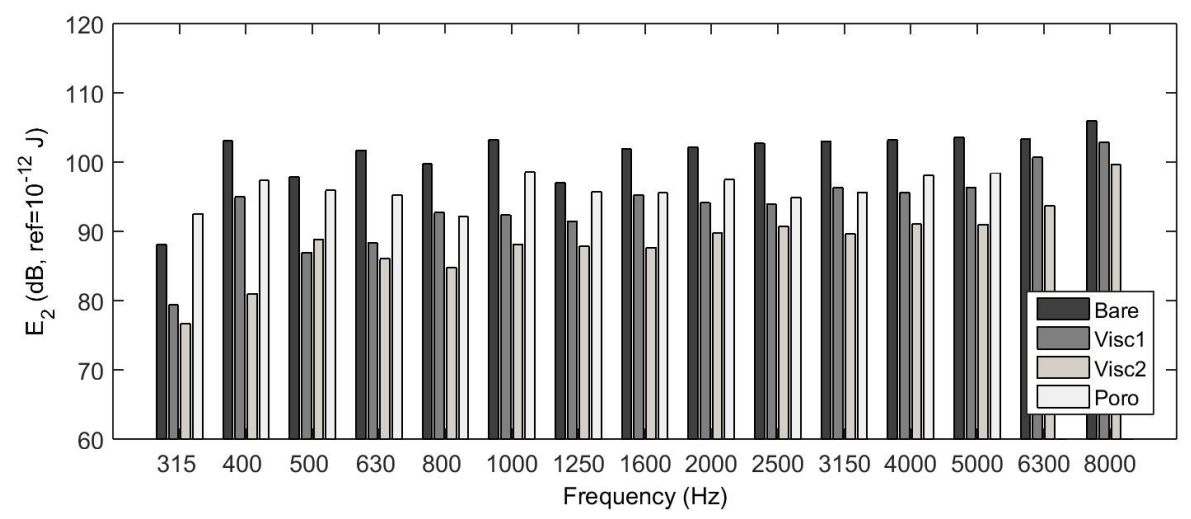

(b)

Figure 14. Subsystem energies in third octave bands for the 4 configurations: (a) Plate energies, (b) Cavity energies

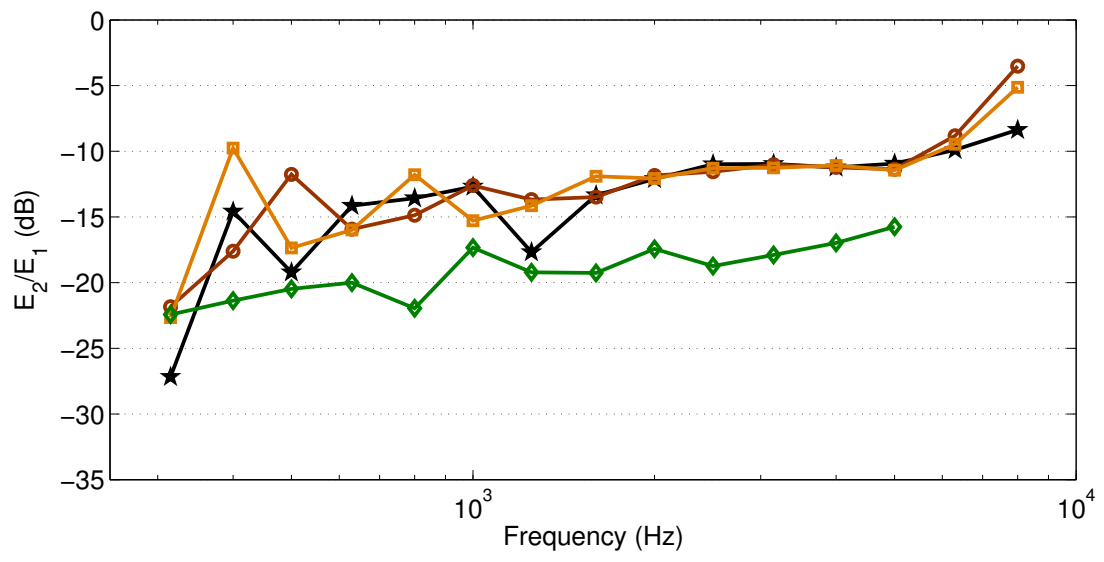

Figure 15. Subsystem energy ratio $E_{2} / E_{1}$ in third octave bands for the 4 configurations: $\star$, Bare configuration; $\square$, Visc1 configuration; $\bigcirc$, Visc2 configuration; $\diamond$, Poro configuration 


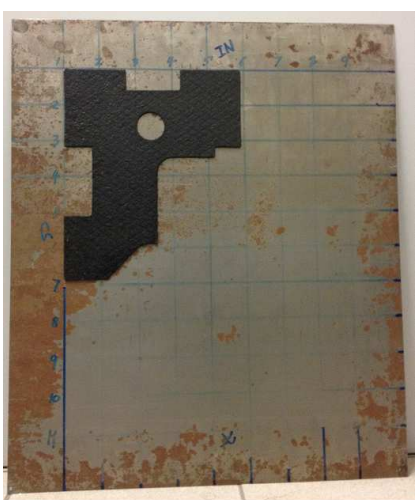

(a)

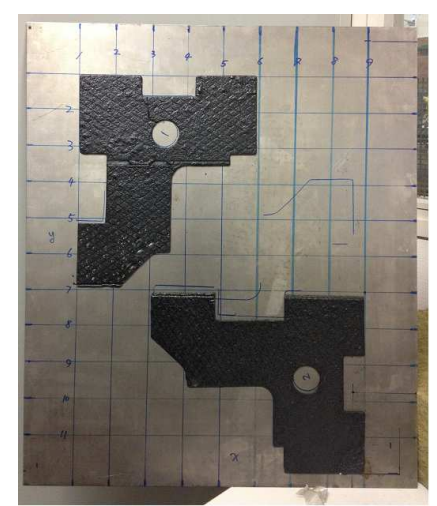

(b)

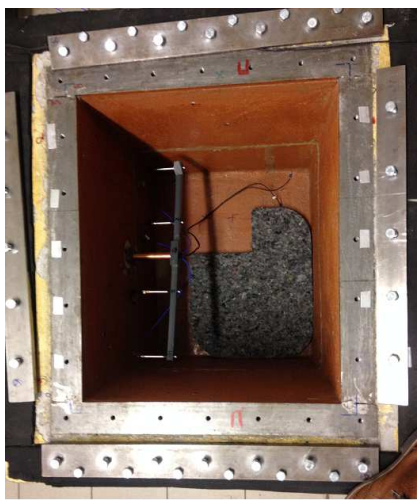

(c)

Figure 16. Pictures of the different treated subsystems used experimentally: (a), plate of the Viscl configuration; (b), plate of the Visc2 configuration; (c), cavity with microphones of the Poro configuration.

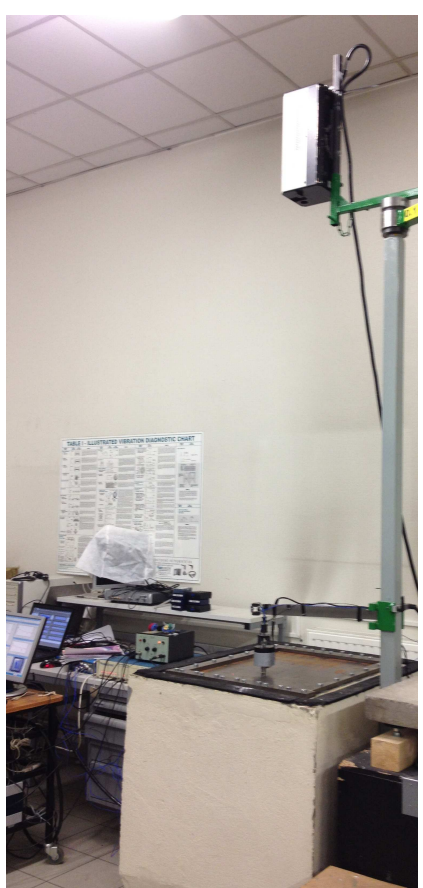

(a)

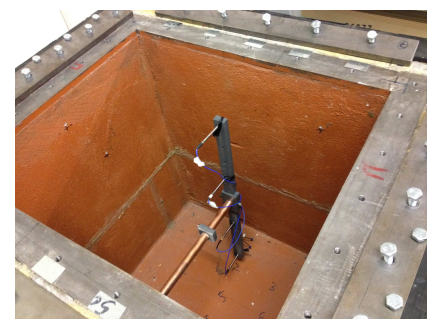

(b)

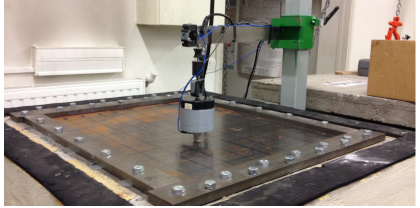

(c)

Figure 17. Experimental setup: (a) Plate-cavity system and the mounted laser vibrometer; (b) Microphones mounted inside the empty cavity; (c) Excitation device. 


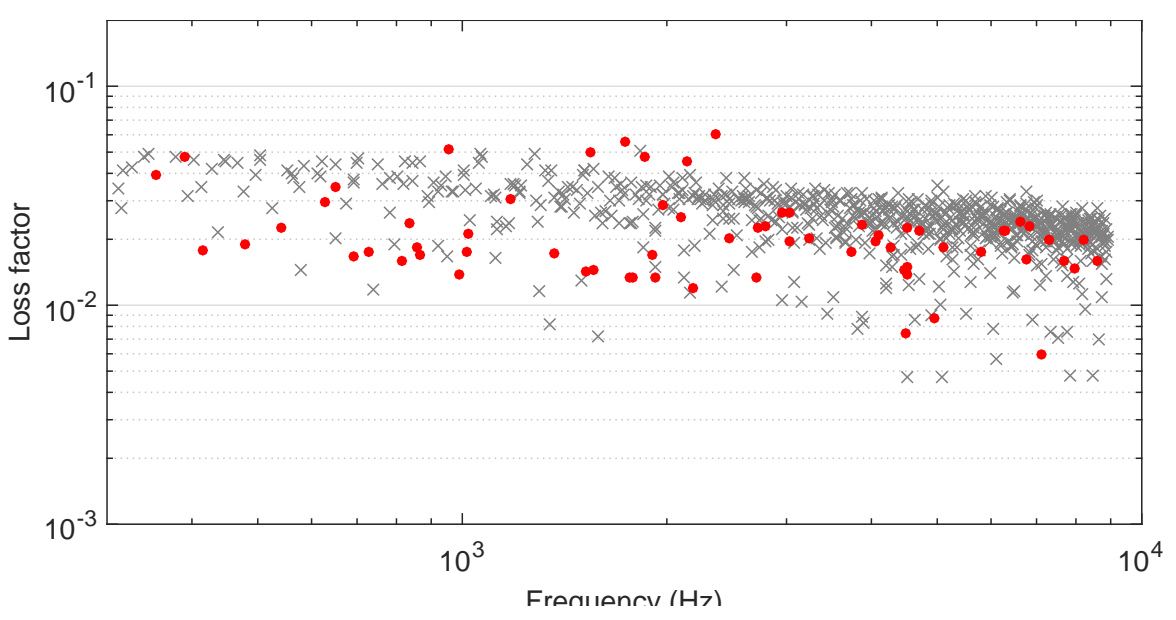

(a)

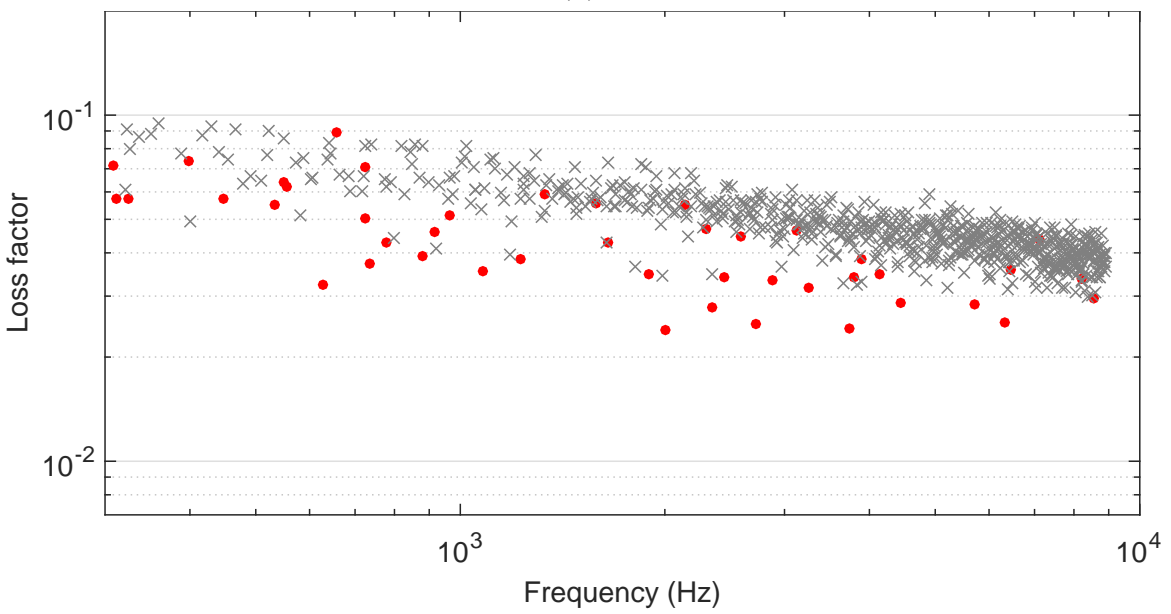

(b)

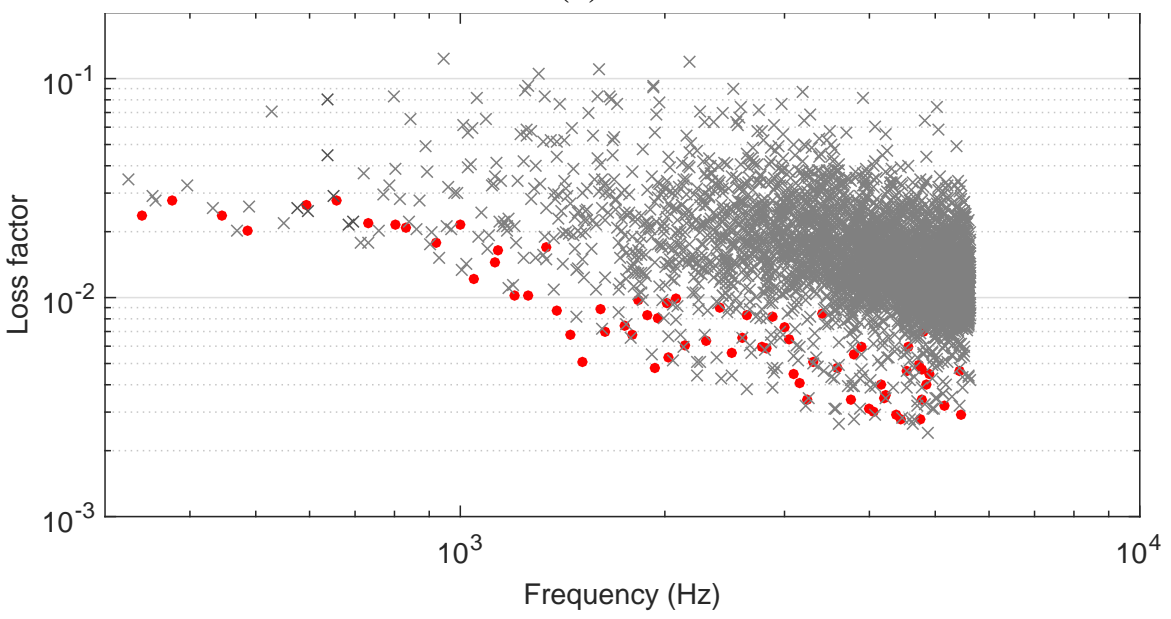

(c)

Figure 18. Modal damping loss factors for the treated subsystems: (a), plate of the Visc1 configuration; (b), plate of the Visc2 configuration; (c), cavity of the Poro configuration. Comparison of two results: $\bullet$, experimental; $\times$, numerical 


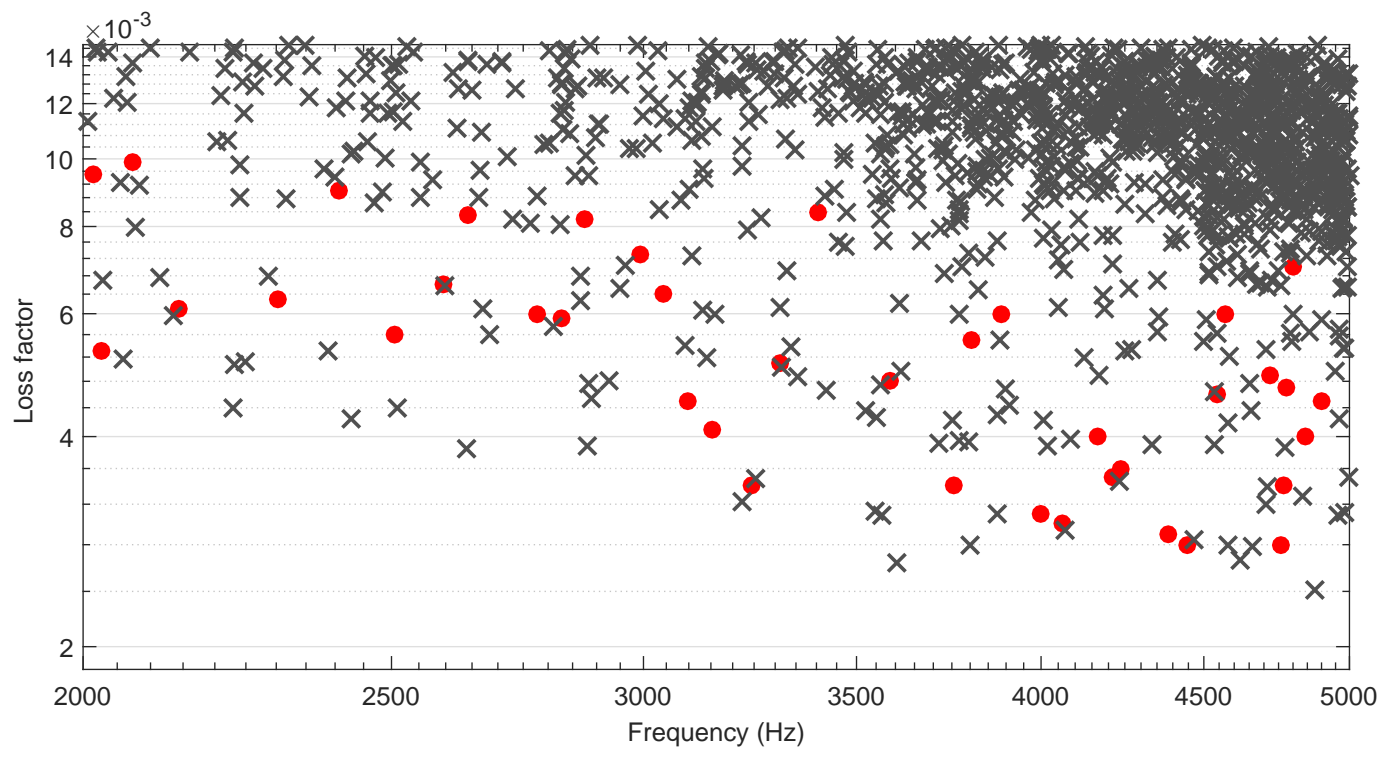

Figure 19. Zoomed view of the high frequency region of Fig. 18c 


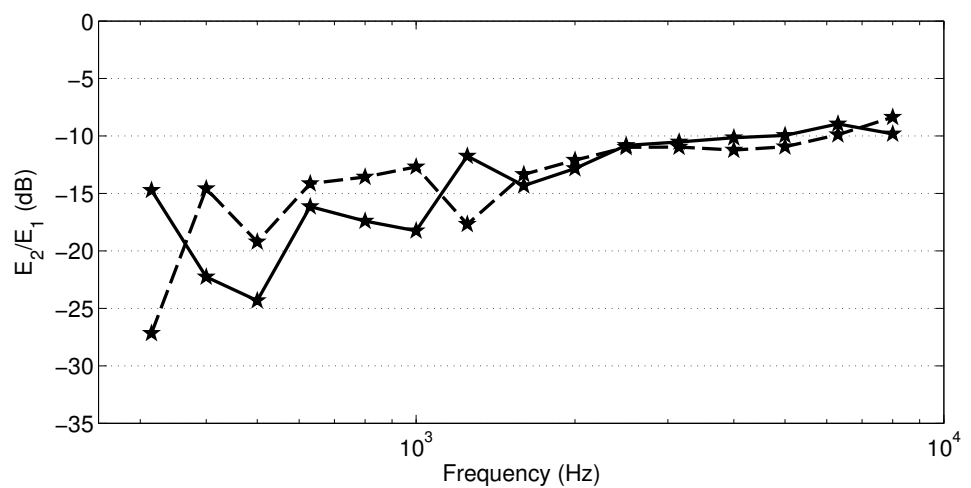

(a)

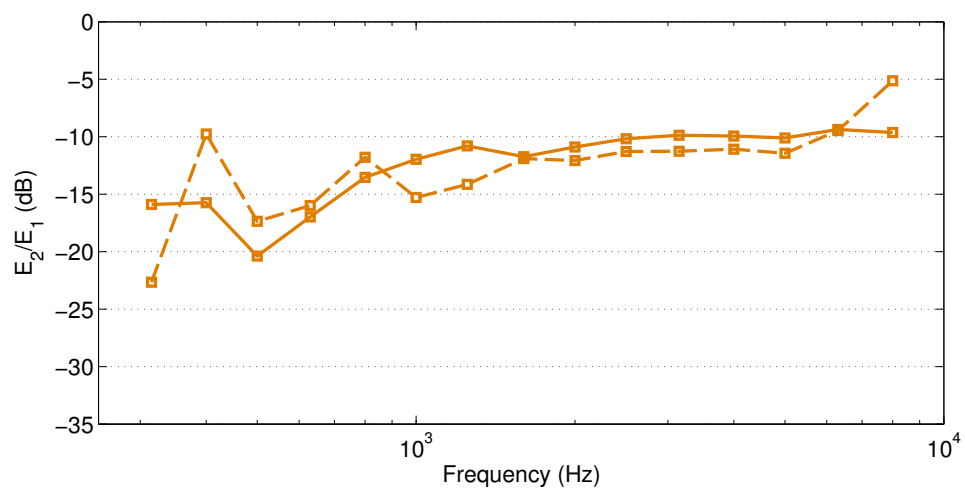

(b)

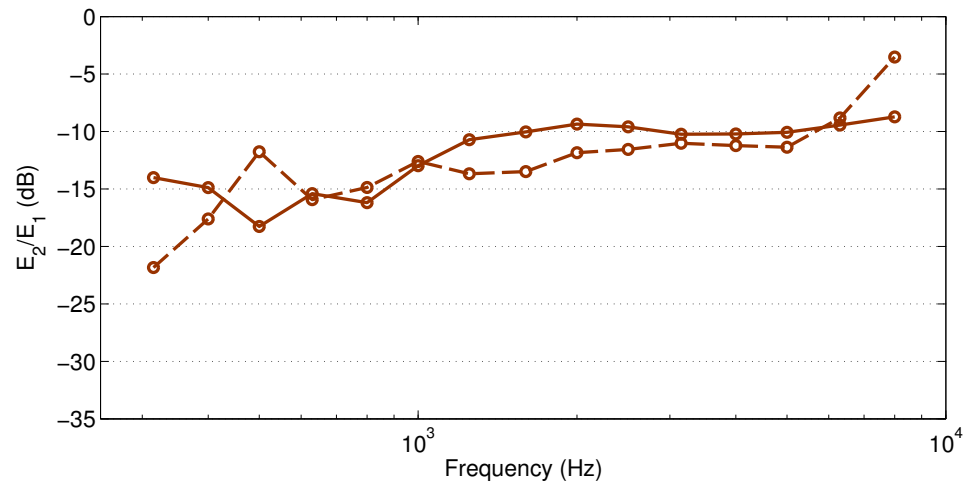

(c)

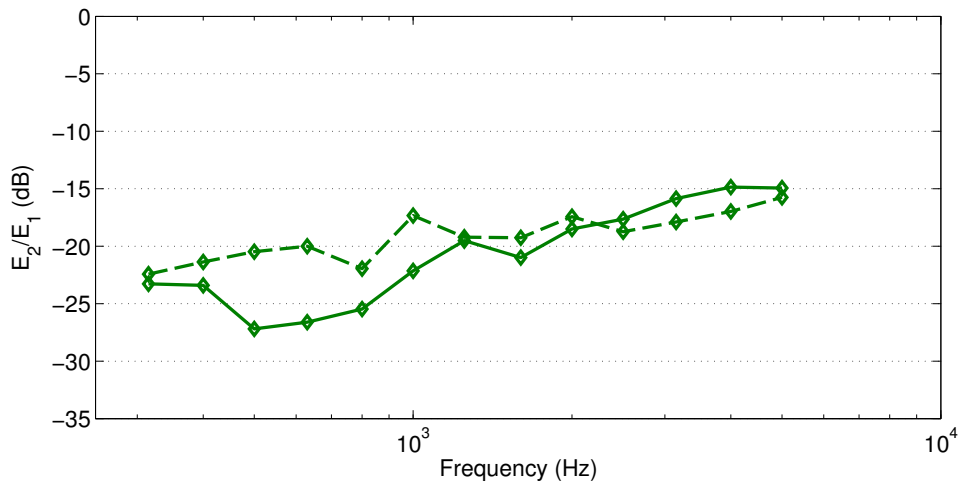

(d)

Figure 20. Subsystem energy ratio in third octave bands for the different configurations: (a), Bare configuration; (b), Visc1 configuration; (c), Visc2 configuration; (d), Poro configuration. Comparison of two results: Solid, experimental; dashed, SmEdA prediction 


\section{Tables}

Table 1

\begin{tabular}{c|ccc}
\hline & Steel & Visco-elastic & Equivalent \\
\hline Thickness $(\mathrm{mm})$ & 1 & 3 & 4 \\
$E(\mathrm{~Pa})$ & $2.03 \mathrm{e} 11$ & $E_{\mathrm{visc}}(f)$ & $E_{\mathrm{eq}, \mathrm{m}}(f)$ \\
$\rho\left(\mathrm{kg} / \mathrm{m}^{2}\right)$ & 7523 & 1533 & 3196 \\
$v$ & 0.33 & 0.35 & 0.34 \\
$\eta$ & $\eta_{\mathrm{ba}}(f)$ & $\eta_{\text {visc }}(f)$ & $\eta_{\mathrm{eq}, \mathrm{m}}(f)$ \\
\hline
\end{tabular}

Mechanical properties of the plate, viscoelastic layer and equivalent plate model. Frequency dependent values given in Fig. 5. (Equivalent parameters computed with MOVISAND software.)

\begin{tabular}{c|cccccccc}
\hline 1/3 octave $(\mathrm{Hz})$ & 315 & 400 & 500 & 630 & 800 & $1 \mathrm{k}$ & $1.25 \mathrm{k}$ & $1.6 \mathrm{k}$ \\
\hline Bare Plate & 6 & 7 & 10 & 11 & 16 & 19 & 24 & 35 \\
Visc1 Plate & 6 & 7 & 9 & 14 & 13 & 21 & 25 & 31 \\
Visc2 Plate & 5 & 6 & 8 & 11 & 15 & 18 & 23 & 30 \\
Empty Cavity & 2 & 3 & 2 & 8 & 14 & 21 & 41 & 90 \\
Poro Cavity & 2 & 3 & 4 & 7 & 14 & 25 & 46 & 82 \\
\hline \hline & $2 \mathrm{k}$ & $2.5 \mathrm{k}$ & $3.15 \mathrm{k}$ & $4 \mathrm{k}$ & $5 \mathrm{k}$ & $6.3 \mathrm{k}$ & $8 \mathrm{k}$ & \\
\hline Bare Plate & 41 & 50 & 69 & 87 & 106 & 144 & 185 & \\
Visc1 Plate & 40 & 60 & 65 & 79 & 105 & 134 & 169 & \\
Visc2 Plate & 37 & 49 & 58 & 76 & 100 & 125 & 160 & \\
Empty Cavity & 151 & 282 & 567 & 1154 & 2096 & 4227 & 8754 & \\
Poro Cavity & 151 & 288 & 543 & 1004 & 1864 & & & \\
\hline
\end{tabular}

Table 2

Number of subsystem modes in third octave bands for each configuration. 